Draft VERSion April 27, 2018

Typeset using LATEX default style in AASTeX61

\title{
THE BLACK HOLE MASSES AND EDDINGTON RATIOS OF TYPE 2 QUASARS
}

\author{
M. Z. KONG ${ }^{1}$ AND LuIS C. $\mathrm{Ho}^{2,3}$
}

\footnotetext{
${ }^{1}$ Department of Physics, Hebei Normal University, No.20 East of South 2nd Ring Road, Shijiazhuang 050024, China

${ }^{2}$ Kavli Institute for Astronomy and Astrophysics, Peking University, Beijing 100871, China

${ }^{3}$ Department of Astronomy, School of Physics, Peking University, Beijing 100871, China
}

\begin{abstract}
Type 2 quasars are an important constituent of active galaxies, possibly representing the evolutionary precursors of traditionally studied type 1 quasars. We characterize the black hole mass $\left(M_{\mathrm{BH}}\right)$ and Eddington ratio $\left(L_{\mathrm{bol}} / L_{\mathrm{Edd}}\right)$ for 669 type 2 quasars selected from the Sloan Digital Sky Survey, using black hole masses estimated from the $M_{\mathrm{BH}}-\sigma_{*}$ relation and bolometric corrections scaled from the extinction-corrected [O III $\lambda 5007$ luminosity. When stellar velocity dispersions cannot be measured directly from the spectra, we estimate them from the core velocity dispersions of the narrow emission lines $[\mathrm{O}$ II] $\lambda \lambda 3726,3729$, [S II] $\lambda \lambda 6716,6731$, and $[\mathrm{O}$ III $] \lambda 5007$, which are shown to trace the gravitational potential of the stars. Energy input from the active nucleus still imparts significant perturbations to the gas kinematics, especially to high-velocity, blueshifted wings. Nonvirial motions in the gas become most noticeable in systems with high Eddington ratios. The black hole masses of our sample of type 2 quasars range from $M_{\mathrm{BH}} \approx 10^{6.5}$ to $10^{10.4} M_{\odot}$ (median $\left.10^{8.2} M_{\odot}\right)$. Type 2 quasars have characteristically large Eddington ratios $\left(L_{\mathrm{bol}} / L_{\mathrm{Edd}} \approx 10^{-2.9}-10^{1.8}\right.$; median $10^{-0.7}$ ), slightly higher than in type 1 quasars of similar redshift; the luminosities of $\sim 20 \%$ of the sample formally exceed the Eddington limit. The high Eddington ratios may be consistent with the notion that obscured quasars evolve into unobscured quasars.
\end{abstract}

Keywords: galaxies: active — galaxies: nuclei — galaxies: Seyfert — quasars: emission lines 


\section{INTRODUCTION}

The classical unified model of active galactic nuclei (AGNs) has served as a useful framework for synthesizing a wide range of observations (Antonucci 1993; Urry \& Padovani 1995; Netzer 2015). In the simplest version of this model, the orientation of the central engine with respect to the viewer plays a major role in determining the observed characteristics, and hence classification, of AGNs. In particular, obscuration along the line-of-sight by a parsec-scale dusty torus dictates whether or not emission from the broad-line region is directly visible: unobscured type 1 sources exhibit broad permitted lines with velocity widths [full width at half maximum (FWHM)] $\gtrsim 1000 \mathrm{~km} \mathrm{~s}^{-1}$, whereas obscured type 2 sources display only narrow emission lines. Within this framework, both types are intrinsically the same.

In recent years, however, it has become increasingly clear that the conventional, purely orientation-based unified model cannot account for the full diversity of the AGN population. Nor is it reasonable that it should. Accretion onto a central supermassive black hole $(\mathrm{BH})$ powers AGNs. To the extent that the general galaxy population certainly evolves strongly with cosmic epoch, so, too, must the population of active galaxies, as BHs coevolve with their hosts (Kormendy \& Ho 2013). The regime of low mass accretion rates (Ho 2008) offers the most dramatic examples of departures from the "standard" paradigm. At the lowest accretion rates, the obscuring torus - indeed, even the broadline region itself - disappears (Elitzur \& Ho 2009; Elitzur et al. 2014). Although less well-studied, "intrinsic" type 2 AGNs also emerge under conditions of higher accretion rates (e.g., Ho et al. 2012; Miniutti et al. 2013; Elitzur \& Netzer 2016; Bianchi et al. 2017; Shu et al. 2017). Furthermore, in the merger-driven picture of quasar evolution (Sanders et al. 1988; Hopkins et al. 2006), dust-obscured (type 2) AGNs arise not simply from geometric viewing angle effects but instead as a direct consequence of a particular evolutionary phase, characterized by different, physically distinct conditions on both large and small scales. In other words, in the evolution-based model type 1 and type 2 AGNs are not intrinsically the same.

The evolutionary scenario linking type 1 and type 2 AGNs should manifest itself most readily in sources of high luminosity. During the past 15 years, increasing attention has been drawn to a population of type 2 quasars, especially the large samples uncovered systematically at optical wavelengths from the Sloan Digital Sky Survey (SDSS). Zakamska et al. (2003) identified 291 objects with redshifts $0.037<z<0.83$ based on standard optical diagnostic emission-line intensity ratios that place them in the category of AGNs. These objects have sufficiently narrow (FWHM $<2000$ $\mathrm{km} \mathrm{s}^{-1}$ ) permitted lines that make them likely candidates for type 2 sources, and, at the same time, about $50 \%$ of them have high enough [O III] $\lambda 5007$ luminosities $\left(>3 \times 10^{8} L_{\odot}\right)$ that, when translated to equivalent $B$-band absolute magnitudes, qualify them as quasars by the historical criterion of $M_{B}<-23$ mag (Schmidt \& Green 1983). Following a similar method, Reyes et al. (2008) expanded the SDSS sample to 887 candidates, the largest catalog to date of optically selected low-to-moderate redshift type 2 quasars ${ }^{1}$. More recently, Alexandroff et al. (2013) further used SDSS to extend the selection of type 2 quasars to $2<z<4.3$.

What are the basic properties of type 2 quasars, and how do they compare with those of the more traditionally studied type 1 quasars? A number of studies suggest that the host galaxies of the two types of quasars indeed appear to differ. Relative to their type 1 counterparts, type 2 quasars seem to have elevated star formation rates (Kim et al. 2006; Zakamska et al. 2008, 2016), a higher incidence of tidal disturbances (Bessiere et al. 2012), and lower halo masses (Ballantyne 2016). Type 2 quasars also have a higher frequency of flat-spectrum radio cores (Lal \& Ho 2010 ).

$\mathrm{BH}$ mass is one of the most fundamental physical parameters for AGNs. Also of interest for understanding the evolutionary state and the interconnections between different AGN populations is their Eddington ratio ${ }^{2}$, which, too, relies on knowledge of the BH mass. Significant progress has been made in developing methods to derive BH masses for type 1 AGNs, through either reverberation mapping of broad emission lines (e.g., Kaspi et al. 2000) or empirically calibrated formalisms that use single-epoch spectra (e.g., Greene \& Ho 2005b; Vestergaard \& Peterson 2006; Ho \& Kim 2015). These techniques, however, cannot be applied to narrow-line AGNs. Apart from a few rare instances in which $\mathrm{BH}$ masses in active galaxies can be measured directly through spatially resolved kinematics (see review in Kormendy \& Ho 2013), in general we must resort to indirect methods to estimate BH masses for type 2 AGNs, principally through the well-known correlations between BH mass and host galaxy bulge stellar mass or velocity dispersion. These empirical relations, of course, were established originally for inactive galaxies at $z \approx 0$ (e.g., Tremaine et al. 2002; Häring \& Rix 2004). When applying these relations to more active systems and especially at higher redshift, we implicitly assume

\footnotetext{
1 Reyes et al. (2008) adopt a slightly modified luminosity criterion of $L_{[\mathrm{O} \text { III }]}>2 \times 10^{8} L_{\odot}$.

${ }^{2}$ The Eddington ratio is defined as $L_{\mathrm{bol}} / L_{\mathrm{Edd}}$, with $L_{\mathrm{bol}}$ the bolometric luminosity and $L_{\mathrm{Edd}}=1.26 \times 10^{38}\left(M_{\mathrm{BH}} / M_{\odot}\right)$ erg s${ }^{-1}$, with $M_{\mathrm{BH}}$ the mass of the $\mathrm{BH}$.
} 
that (1) AGNs obey the same empirical scaling relations as local inactive galaxies, (2) these relations do not evolve with redshift, or (3) any potential redshift dependence can be corrected.

In this paper, we investigate the possibility of estimating BH masses for type 2 quasars using the $M_{\mathrm{BH}}-\sigma_{*}$ relation (Ferrarese \& Merritt 2000; Gebhardt et al. 2000). We proceed in two steps. Using the SDSS-selected sample of type 2 quasars from Reyes et al. (2008), we directly measure $\sigma_{*}$ for a subset of sources having spectra of sufficiently high quality with detectable stellar features. For the majority of the sample for which these conditions could not be met, we estimate $\sigma_{*}$ indirectly from the widths of their nebular emission lines. In AGNs of lower luminosity, it has been well-established that the kinematics of the ionized gas in the narrow-line region generally trace the virial motions of the stars of the host galaxy bulge (e.g., Whittle 1992; Nelson \& Whittle 1996). In particular, in Seyfert galaxies the velocity width of [O III $\lambda 5007$ traces $\sigma_{*}$, albeit with considerable scatter. Nelson (2000) found that $\sigma_{g}$, the velocity dispersion derived from ionized gas, can be used as a proxy to study the $M_{\mathrm{BH}}-\sigma_{*}$ relation of AGNs. Greene \& Ho (2005a) investigated the relation between gaseous and stellar kinematics for a large sample of SDSS-selected type 2 AGNs, showing that $\sigma_{g}$ correlates best with $\sigma_{*}$ when the gas velocities are derived from the core of the [O III] line, after removing its asymmetric wings. The $\sigma_{g}-\sigma_{*}$ relation also holds for the lower ionization lines [O II] $\lambda \lambda 3726,3729$ and [S II] $\lambda \lambda 6716,6731$. The analysis of Greene \& Ho (2005a) focused on AGNs of relatively low luminosity, with $L_{[\mathrm{O} \text { III }]} \lesssim 10^{8} L_{\odot}$. Ho (2009) extended these results to sources of even lower luminosity, using [N II] $\lambda 6583$.

Is the $\sigma_{g}-\sigma_{*}$ relation applicable to the more powerful type 2 quasars? As the AGN luminosity increases, the narrowline region gas may be increasingly susceptible to non-gravitational perturbations (e.g., radiation pressure), and it is unclear the extent to which the kinematics of the ionized gas still track the virial velocity of the underlying stellar gravitational potential. Greene et al. (2009) analyzed a sample of 111 type 2 quasars and found that the line widths of [O II] and [O III] exhibit no significant correlation with $\sigma_{*}$. This suggests that $\sigma_{g}$ cannot be used to estimate $\mathrm{BH}$ mass for AGNs with luminosities in the range of quasars.

We investigate this problem with a new, independent analysis of the SDSS-selected sample of type 2 quasars of Reyes et al. (2008), taking special care to account for factors that may influence $\sigma_{g}$, such as asymmetries and other substructure in the line profile. Although the scatter is considerable, we find that, as in lower-luminosity AGNs, $\sigma_{g}$ does still trace $\sigma_{*}$ usefully in type 2 quasars. We use our newly derived $\sigma_{g}-\sigma_{*}$ relation, in combination with the latest $M_{\mathrm{BH}}-\sigma_{*}$ relation, to estimate $\mathrm{BH}$ masses and Eddington ratios for the Reyes et al. (2008) sample of low-redshift type 2 quasars.

This paper is organized as follows. Section 2 discusses the sample selection. Section 3 presents measurements of $\sigma_{*}$ and the analysis of the emission lines to derive $\sigma_{g}$. Section 4 compares $\sigma_{g}$ and $\sigma_{*}$, discusses the prevalence of novirial motions in the narrow-line region, and applies the velocity dispersions to estimate $\mathrm{BH}$ masses and Eddington ratios. We summarize our main conclusions in Section 5. We adopt the following cosmological parameters: $\Omega_{m}=0.3, \Omega_{\Lambda}=$ 0.7 , and $H_{0}=70 \mathrm{~km} \mathrm{~s}^{-1} \mathrm{Mpc}^{-1}$.

\section{SAMPLE}

As one of the main purposes of our work is to measure stellar velocity dispersions and compare them with velocity dispersions derived from ionized gas, we need access to the rest-frame optical stellar continuum and a variety of wellmeasured strong emission lines. Thus, we focus on the sample of 887 type 2 quasars with redshifts $0.037<z<0.83$ from Reyes et al. (2008). The relatively low redshifts of the sample also help to minimize possible redshift evolution of the $M_{\mathrm{BH}}-\sigma_{*}$ relation, an assumption critical to our application of the velocity dispersions to estimate $\mathrm{BH}$ masses for the sample.

We obtain the optical spectra of the sample from the seventh data release of SDSS (Abazajian et al. 2009). Some of the objects exhibit obvious double-peaked or otherwise conspicuous complex substructure in their emission-line core. The Reyes et al. sample contains a total of 126 sources with complex line substructure, among them 42 that have unambiguous double-peaked profiles. The line substructure is most evident in [O III], presumably because of its high signal-to-noise ratio $(\mathrm{S} / \mathrm{N})$, but in many cases it can also be seen in the weaker low-ionization lines. The objects with complex profile substructure span a similar range of [O III] luminosity and redshift as the parent sample (Figure 1). A variety of physical origins may give rise to kinematic complexity in the narrow-line gas. For instance, double-peaked profiles may be indicative of galaxy mergers, large-scale disk rotation, or outflows (e.g., Liu et al. 2010; Ge et al. 2012). The interaction of compact radio jets with the interstellar medium of the host galaxy can produce flat-topped cores and other profile substructure (e.g., Whittle et al. 1988; Gelderman \& Whittle 1994). Whatever the case may be, these complexities will complicate the interpretation of the line widths and compromise our ability to use them to 
trace virial velocities. We omit the 126 sources with complex profiles from our analysis of the emission-line widths, but we retain them for the purposes of measuring stellar velocity dispersions.

\section{ANALYSIS}

\subsection{Stellar Velocity Dispersions}

\subsubsection{Method}

The basic principle for measuring the line-of-sight stellar velocity dispersion of a galaxy through integrated spectroscopy is to estimate the degree to which the intrinsic stellar spectrum of the galaxy has been broadened by the internal motions (velocity dispersion) of its constituent stars. Traditional measurements are mostly based on Fourier or cross-correlation techniques (e.g., Sargent et al. 1977; Tonry \& Davis 1979; Bender 1990), but more modern studies increasingly favor methods based on direct fitting of the spectra in pixel space (e.g., Kelson et al. 2000; Barth et al. 2002; Cappellari \& Emsellem 2004; Greene \& Ho 2006, hereafter GH06). One of the advantages of the latter method is that it is straightforward to exclude emission lines and other unwanted or bad pixels from the fit. The robustness of the fit can be evaluated readily from direct comparison of the broadened template spectrum with the galaxy spectrum.

We use the publicly available penalized pixel-fitting code pPXF of Cappellari \& Emsellem (2004) to measure $\sigma_{*}$. Prior to analysis, we correct the SDSS spectra for foreground dust reddening, using the extinction values of Schlafly \& Finkbeiner (2011) and the Galactic extinction curve of Cardelli et al. (1989). The spectra were then shifted to their rest frame, based on SDSS-derived redshifts. Our adopted values for the $V$-band Galactic extinction and redshift are listed in Table 1.

The pPXF program models the galaxy spectrum as

$$
M_{\text {mod }}(x)=P(x)\left\{\sum_{j=1}^{N} w_{j}\left[T_{j}(x) \otimes G(x)\right]\right\}+C(x),
$$

where $w_{j}$ is the fractional contribution of the $j$ th stellar template $T_{j}(x)$ to the model flux, $\otimes$ denotes convolution, $G(x)$ is a Gaussian broadening function with dispersion $\sigma_{m}$, and $P(x)$ is a Legendre polynomial that accounts for potential mismatches in shape between the galaxy and the templates, which might arise, for instance, from internal reddening, mismatches in stellar population, or residual calibration errors (e.g., GH06; Liu et al. 2009). The term $C(x)$ is a Legendre polynomial that represents any additional additive contribution, such as a power-law continuum from scattered light from the central AGN or a hot continuum from young $\mathrm{O}$ and B stars. The best-fit parameters are obtained by minimization of $\chi^{2}$ using the nonlinear Levenberg-Marquardt algorithm implemented in the IDL package mpfit $^{3}$ (Markwardt 2009).

The template $T(x)$ in principle should faithfully match the stellar population of the galaxy. However, in practice, it can be reasonably well represented by the spectrum of an optimally weighted linear combination of several stars or of even a well-chosen single star (e.g., Barth et al. 2002; GH06; Ho et al. 2009). There are many available libraries of stellar templates (e.g., Bruzual \& Charlot 2003; Valdes et al. 2004; Sánchez-Blázquez et al. 2006), which have different wavelength coverage and spectra resolution. In light of the instrumental spectral resolution of SDSS (mean FWHM $=2.94 \pm 0.31 \AA$ over the region $4100-5400 \AA$ used for our analysis of $\sigma_{*}$; see below), the most suitable choice for our applications is the Indo-U.S. stellar spectral library (Valdes et al. 2004), which covers the wavelength range 3460-9464 $\AA$ at a spectral resolution of FWHM $=1.35 \AA$ (Beifiori et al. 2011). Consistent with prior studies, a weighted linear combination of late-type (F, G, K, and M) red giant (luminosity class III) stars of near-solar metallicity provides a good match to the stellar continuum of the host galaxies. We also include an A-type dwarf (luminosity class V) star to account for those galaxies that have a substantial post-starburst stellar population.

As the template stars used in our fits were observed with a substantially higher spectral resolution than the SDSS science targets, the model velocity dispersion $\left(\sigma_{m}\right)$ needs to be corrected to yield the intrinsic stellar velocity dispersion $\left(\sigma_{*}\right)$ of the galaxy. We adopt $\sigma_{*}^{2}=\sigma_{m}^{2}+\sigma_{\text {inst }, \mathrm{V}}^{2}-\left(\sigma_{\text {inst }, \mathrm{S}} /(1+z)\right)^{2}$, where the instrumental resolution is $\sigma_{\text {inst }, \mathrm{V}}=0.57$ $\AA$ for the Valdes et al. (2014) templates and $\sigma_{\text {inst,S }}=1.25 \AA$ for the SDSS spectra.

Careful consideration should be given to the choice of wavelength range over which to perform the fit, with the goal of avoiding regions contaminated by strong emission lines and other known sources of systematic bias. At optical

\footnotetext{
${ }^{3}$ http://purl.com/net/mpfit
} 
wavelengths, the Ca II $\lambda \lambda 8498,8542,8662$ infrared triplets are optimal for stellar dynamical work because of their relative insensitivity to stellar population (Dressler 1984) and AGN contamination (GH06), but they are shifted out of the SDSS bandpass and inaccessible to most of our sample. We do not consider $\mathrm{Ca} \mathrm{H}+\mathrm{K} \lambda \lambda 3934,3968$ because they are blended with [Ne III] $\lambda 3968$ and $\mathrm{H} \epsilon \lambda 3970$ (GH06), the latter being especially problematic when a strong A-star population is present. After much experimentation, we finally adopt $4100-5400 \AA$ as the fitting region, but taking care to avoid strong emission lines (He II $\lambda 4686, \mathrm{H} \beta \lambda 4861$, [O III] $\lambda \lambda 4959,5007$ ). We exclude the Mg Ib $\lambda \lambda 5167,5173,5184$ triplets, which suffer from potential systematic effects due to $[\mathrm{Mg} / \mathrm{Fe}]$ enhancement (Barth et al. 2002), but do include the $\mathrm{G}$ band at $4304 \AA$, after masking out $\mathrm{H} \gamma \lambda 4340$ and [O III] $\lambda 4363$. Figure 2 shows a sample fit. Our chosen fitting regions are similar to those of Greene et al. (2009).

Kelson et al. (2000) found that $\sigma_{*}$ is not very sensitive to $P(x)$ and $C(x)$, with differences of less than $1 \%$ in $\sigma_{*}$ for Legendre polynomials of different orders. By contrast, similar tests by GH06 and Barth et al. (2002) indicate greater sensitivity of $\sigma_{*}$ to the choice of polynomial order, particularly for fits performed over a relatively narrow wavelength range. Our own tests with the PPXF code confirm that the exact choice of polynomial order does not have a significant impact on $\sigma_{*}$ (generally $\lesssim 2 \%-5 \%$ ), so long as we avoid very low or high values. As a compromise, and for concreteness, we choose a third-order polynomial for $P(x)$ and $C(x)$.

To verify the robustness of our fitting method, we measured $\sigma_{*}$ for the sample of 504 SDSS type 2 AGNs originally used by GH06 (their Figure 2) and performed a direct comparison between the two sets of measurements. The agreement is excellent (Figure 3). Defining the difference between the two sets of independent measurements by $\Delta \sigma_{*} \equiv \log \sigma_{*}$ (ours) $-\log \sigma_{*}$ (GH06), we find a mean value of $\left\langle\Delta \sigma_{*}\right\rangle=0.002 \pm 0.05$, with little evidence for any systematic dependence on $\sigma_{*}$.

\subsubsection{Error Estimates}

The pPXF code returns formal $(1 \sigma)$ errors of $\sim 13 \% \pm 6 \%$ on $\sigma_{*}$ for spectra with S/N greater than 15 (Cappellari \& Emsellem 2004). However, in practice, errors on $\sigma_{*}$ may be dominated by template mismatch or other sources of systematic uncertainties, especially for spectra of AGN host galaxies whose continuum may be contaminated by other sources of emission. The situation for type 2 AGNs is more favorable than that for type 1 AGNs (e.g., GH06), but even type 2 AGNs are not immune. For instance, the equivalent widths (EWs) of the stellar features may be diluted by a featureless continuum from scattered light from the nucleus (e.g., Liu et al. 2009; Alexandroff et al. 2013).

We perform a series of Monte Carlo simulations to evaluate the impact of template mismatch. We choose high-S/N ( $~ 90$ per pixel near the G band) SDSS spectra of six inactive galaxies with $\sigma_{*}=85,114,125,178,220$, and $360 \mathrm{~km}$ $\mathrm{s}^{-1}$, roughly spanning the full range of $\sigma_{*}$ encountered in our sample of type 2 quasars. In this study, we define the G band over $4285-4328 \AA$, with the continuum set to the midpoint of a power law connecting these two end points. The $\mathrm{S} / \mathrm{N}$ is evaluated in the region $4400-4600 \AA$, which is relatively free from strong emission or absorption lines. We fit each spectrum $10^{4}$ times, each trial randomly varying the set of five template stars (A-type dwarf plus a red giant of type F, G, K, and M) drawn from the Indo-U.S. stellar template library of Valdes et al. (2004). Figure 4 shows that the uncertainties on $\sigma_{*}$ are always small, $\sim 4 \% \pm 1 \%$, with no systematic dependence on $\sigma_{*}$.

The S/N of the spectrum has a larger effect on $\sigma_{*}$ measurements (Cappellari \& Emsellem 2004). The strength (EW) of the stellar absorption lines will also matter. Furthermore, even for spectra of the same $\mathrm{S} / \mathrm{N}$ and absorption-line EW, we anticipate that there may be systematic biases as a function of $\sigma_{*}$ : for the same EW, a larger $\sigma_{*}$ results in a broader, shallower spectral feature, which is more adversely affected by noise fluctuations and potential dilution by AGN continuum. We investigate these effects as follows. The above-described set of six high-S/N spectra of inactive galaxies have $\mathrm{EW}(\mathrm{G}$ band $) \approx 10 \AA$. We dilute the $\mathrm{G}$ band from the original strength of $\mathrm{EW} \approx 10 \AA$ to $1 \AA$, in steps of $1 \AA$, by artificially adding a constant continuum level to the spectrum. Poisson noise is introduced to produce a series of spectra with $\mathrm{S} / \mathrm{N} \approx 3-40$, in steps of 1 when $\mathrm{S} / \mathrm{N}<10$, and in steps of 2 when $10 \leq \mathrm{S} / \mathrm{N} \leq 40$. In total, a grid of 230 pairs of $\mathrm{EW}$ and $\mathrm{S} / \mathrm{N}$ was created for each of the six original input spectra, and for each pair we simulate 500 realizations.

Figure 5 summarizes the results of these simulations. As expected intuitively, the fractional error on $\sigma_{*},\left\langle\delta \sigma_{*}\right\rangle \equiv$ $\left\langle\left[\sigma_{*}\right.\right.$ (input) $-\sigma_{*}$ (output) $] / \sigma_{*}$ (input $\left.)\right\rangle$, becomes enormous when both S/N and EW are low. On the other hand, $\left\langle\delta \sigma_{*}\right\rangle \lesssim$ $15 \%-20 \%$ when $\mathrm{EW} \gtrsim 6 \AA$ and $\mathrm{S} / \mathrm{N} \gtrsim 10$. The dependence on $\sigma_{*}$ is weak, especially when $\sigma_{*} \gtrsim 200 \mathrm{~km} \mathrm{~s}^{-1}$. We utilize the results of Figure 5 to empirically estimate the systematic uncertainty for our actual $\sigma_{*}$ measurements. The final error budget for $\sigma_{*}$ (Table 1) is the quadrature sum of three components: (1) the formal statistical uncertainty from 
pPXF, (2) the systematic uncertainty due to potential template mismatch, which, based on the experiments described above, we assume to be $4 \%$, and (3) the statistical uncertainty due to $\mathrm{S} / \mathrm{N}$ and EW.

\subsection{Gaseous Velocity Dispersions}

A principal goal of this study is to evaluate whether and how well the velocity dispersion of the ionized gas in the narrow-line region of type 2 quasars traces the velocity dispersion of the stars in their host galaxies. Prior to measuring the emission lines, the stellar continuum should be properly modeled and subtracted, following essentially the same procedure described in Section 3 (see also Ho et al. 1997a). The main modification is that the wavelength range of the fit now extends from $\sim 3700$ to $7000 \AA$ in order to cover [O II] $\lambda \lambda 3726,3729$, [O III] $\lambda \lambda 4959,5007$, and [S II] $\lambda \lambda 6716,6731$, the set of lines we use to measure the gaseous velocity dispersion $\left(\sigma_{g}\right)$. This template fitting method provides a first-order estimate of the global continuum, but it is unlikely to achieve a high enough accuracy to yield a robust determination of the local continuum for all the emission lines of interest. We therefore sometimes need to apply a second-order correction to adjust the local continuum during the process of fitting the individual lines.

The narrow emission lines of AGNs generally have complex shapes that cannot be well described by a single Gaussian function. The profiles of most narrow lines, especially higher ionization lines such as [O III], often have an asymmetric blue, and at times red, wing (e.g., Whittle 1985; Veilleux 1991). Our sample of type 2 quasars exhibits similar trends. Although the profile asymmetry is most obvious for [O III] (most likely because of its high $\mathrm{S} / \mathrm{N}$ ), it is also frequently discernible for the lower ionization lines of $[\mathrm{O}$ II] and [S II] when the spectra have sufficiently high S/N. Following standard practice (e.g., Ho et al. 1997b; Greene \& Ho 2005a), we fit ${ }^{4}$ each line with a single Gaussian component to represent the "core" $\left(\sigma_{g}^{c}\right)$, and, if statistically warranted, we add another Gaussian component to reproduce the "wing" of the line. Some examples are shown in Figure 6. The wavelength separations of the three doublets are fixed to their laboratory values. We force the components of each doublet to have the same velocity profile, even though in actuality each of the two transitions of the [O II] and [S II] doublets has slightly different critical densities and hence can have mildly different velocity widths (Filippenko \& Halpern 1984; Ho et al. 1996). We fix the flux ratio of [O III] $\lambda 5007 /[\mathrm{O}$ III] $\lambda 4959$ to the theoretical value of 3, but for the two densitometers, [O II] $\lambda 3729 /[\mathrm{O}$ II] $\lambda 3726$ is allowed to vary from 0.3 to 1.5 , and [S II] $\lambda 6716 /[\mathrm{S} \mathrm{II}] \lambda 6731$ can range between 0.45 and 1.5 (Osterbrock 1989).

Apart from the emission-line widths derived from the above-described parametric fits, we also provide a simpler estimate of line width from a direct, non-parametric measurement of the intrinsic FWHM (i.e. after correction for instrumental resolution) of the line profile itself. Then, the Gaussian-equivalent velocity dispersion is $\sigma_{m} \equiv$ FWHM/2.35. Instead of measuring FWHM directly from the data, which is affected by noise, it is more effective to calculate it from a model (noise-free) representation of the line profile. We construct the model by decomposing the original profile using a series of Gaussians, and then summing up the individual components. We use as many Gaussians as statistically necessary to fit the profile. In general two Gaussians are sufficient for each of the doublet lines of [O II] and [S II], but [O III] may occasionally require up to four to properly account for more extended wings. There is no physical meaning attached to any of the Gaussian components; they just serve as a convenient representation of the data. Lastly, as in Greene \& Ho (2005a), we compute the second moment $\left(\sigma_{g}^{s}\right)$ of the best-fit model to describe the width of the overall global line profile.

The core velocity dispersions and second moments for [O II], [S II], and [O III], corrected for the instrumental resolution of $\mathrm{SDSS}^{5}$, are listed in Table 1. The error budget for $\sigma_{g}^{c}$ consists of three terms combined in quadrature: (1) a formal, statistical uncertainty (generally $\lesssim 5 \%$ ) from mpfit; (2) a systematic uncertainty due to errors in the placement of the local continuum, and (3) a systematic uncertainty due to the choice of initial parameters for the multi-component fit. We estimate terms (2) and (3) through a series of Monte Carlo simulations by changing the initial fitting parameters. The error budget for $\sigma_{g}^{s}$ includes only terms (2) and (3).

\subsection{External Comparison}

Greene et al. (2009) performed an independent analysis of the Reyes et al. (2008) sample, using a methodology very similar that employed in this work. They provide $\sigma_{*}$ measurements for 111 objects, as well as FWHM values for $[\mathrm{O}$ II] and [O III]. Figure 7 directly compares our measurements with those of Greene et al. (2009). The agreement is reasonably good in the mean, although the scatter is relatively large for $\sigma_{*}$. The mean difference between the two sets

\footnotetext{
4 The fits are performed using the IDL code mpfit.

5 The average instrumental spectral resolution of SDSS for our sample is FWHM $=2.54 \pm 0.30 \AA$ for [O II], $3.03 \pm 0.35 \AA$ for [O III], and $3.24 \pm 0.39 \AA$ for $[\mathrm{S}$ II] .
} 
of measurements $\left\langle\Delta \sigma_{*}\right\rangle=-0.072 \pm 0.24$, where $\Delta \sigma_{*} \equiv \log \sigma_{*}$ (ours) $-\log \sigma_{*}$ (Greene +09$)$. As for the emission-line widths, $\langle\Delta \mathrm{FWHM}\rangle=0.012 \pm 0.04$ for $[\mathrm{O}$ II] and $-0.002 \pm 0.03$ for [O III]

\subsection{Final Set of Measurements}

Out of the original sample of 887 sources, $449(51 \%)$ have spectra with $\mathrm{S} / \mathrm{N}>3$ per pixel and detectable $\mathrm{G}$ band with EW larger than $3 \AA$. While we succeeded to measure $\sigma_{*}$ for most of these, many of the uncertainties are rather large. For our subsequent analysis, we only make use of the subset of objects with $\sigma_{*}$ measurements that are clearly in excess of the SDSS spectral resolution $\left(\sim 70 \mathrm{~km} \mathrm{~s}^{-1}\right)$ and that have fractional uncertainties $\leq 20 \%$. Only 219 objects ( $25 \%$ of the original sample) satisfy these criteria. The number of $\sigma_{*}$ measurements increases to 281 if we relax the fractional uncertainty to $30 \%$. As for the gaseous line widths, after excluding the 126 objects with kinematically complex core structure, 594 of the remaining 761 objects have a $\sigma_{g}^{c}$ measurement with an intrinsic value larger than $70 \mathrm{~km} \mathrm{~s}^{-1}$ and a fractional uncertainty $\leq 20 \%$ for at least one of the three principal emission lines ([O II], [O III], and [S II]). The number of $\sigma_{g}^{c}$ measurements increases to 697 if the fractional uncertainty is relaxed to $30 \%$. The majority of the sample has useful measurements of $\sigma_{g}^{s}$, at least for [O III], the strongest line, but often also for [O II] and [S II]. The final set of velocity measurements is given in Table 1 . We only list measurements with fractional uncertainties less than $30 \%$.

In total, 142 objects have measurements with fractional uncertainties $\leq 20 \%$ for both $\sigma_{*}$ and $\sigma_{g}^{c}$ for at least one emission line. Not surprisingly, they are drawn predominantly from the portion of the parent sample with the lowest luminosities (median $L_{[\mathrm{O} \mathrm{III}]} \approx 10^{42.5} \mathrm{erg} \mathrm{s}^{-1}$ ) and redshifts (median $z \approx 0.2$ ), although on average they are still more distant and much more luminous than the sample of type 2 AGNs of Greene \& Ho (2005a; Figure 1).

\section{RESULTS}

\subsection{Comparison Between Velocity Dispersions of Stars and Ionized Gas}

Figure 8 begins with a comparison between $\sigma_{*}$ and $\sigma_{g}$, defined simply as FWHM/2.35 (appropriate for a Gaussian function), where FWHM is measured from the total line profile of [O II] (166 objects), [S II] (154 objects), and [O III] (169 objects). It is clear that most of the gas velocities are significantly larger than the stellar velocities, and there is little correlation between the two quantities. The average value and standard deviation of $\left\langle(\mathrm{FWHM} / 2.35) / \sigma_{*}\right\rangle$ is $1.17 \pm 0.38,1.15 \pm 0.35$, and $1.11 \pm 0.40$ for [O II], [S II], and [O III]. This is consistent with the conclusion of Greene et al. (2009) and Liu et al. (2009), who parameterized the gas velocity dispersion in the same way. The situation is even more extreme for $\sigma_{g}^{s}$, the second moment of the line, which is maximally sensitive to the wings of the profile. Figure 9a shows that $\sigma_{g}^{s} \gtrsim \sigma_{*}$ for nearly all the type 2 quasars, some by very significant amounts. The range of $\sigma_{g}^{s} / \sigma_{*}$ spans 0.65 to 6.01 , with $\left\langle\sigma_{g}^{s} / \sigma_{*}\right\rangle=1.73 \pm 0.75,1.66 \pm 0.80$, and $1.85 \pm 0.88$ for [O II], [S II], and [O III], respectively. In other words, the narrow lines of type 2 quasars invariably have highly non-Gaussian, extended, usually blue asymmetric wings. Note that for lower-luminosity AGNs, only the high-ionization line [O III] shows blue asymmetry (Greene \& Ho 2005a; Ho 2009; Figure 9a).

By contrast, once we remove the line wings and focus only on the core of the emission-line profile $\left(\sigma_{g}^{c}\right)$, the situation improves dramatically (Figure $9 \mathrm{~b}$ ). The gas velocities become comparable to the stellar velocities, and the scatter decreases. We find $\left\langle\sigma_{g}^{c} / \sigma_{*}\right\rangle=1.06 \pm 0.32,0.99 \pm 0.32$, and $1.00 \pm 0.36$ for [O II], [S II], and [O III]. Because of the small sample size, limited dynamic range, and large measurement uncertainties, the correlation between $\sigma_{*}$ and $\sigma_{g}^{c}$ is not statistically strong for the type 2 quasars alone. The Spearman's rank correlation coefficients are $\rho=0.32$ and 0.34 for $[\mathrm{O} \mathrm{II}]$ and $[\mathrm{S} \mathrm{II}]$, respectively, with a probability for the null hypothesis of no correlation of $P_{\text {null }} \approx 0.02$; for [O III], $\rho=0.17$ and $P_{\text {null }}=0.1$. The lower luminosity sources of Greene \& Ho $(2005 \mathrm{a})^{6}$ behave very similarly: $\left\langle\sigma_{g}^{c} / \sigma_{*}\right\rangle=1.13 \pm 0.38,1.11 \pm 0.35$, and $1.01 \pm 0.35$. For both samples combined, we obtain $\left\langle\sigma_{g}^{c} / \sigma_{*}\right\rangle=1.13 \pm 0.38$, $1.11 \pm 0.35$, and $1.00 \pm 0.35$. Now the $\sigma_{*}-\sigma_{g}^{c}$ relation is highly statistically significant, with $\rho=0.43,0.46$, and 0.42 and $P_{\text {null }}<10^{-6}$ for $[\mathrm{O} \mathrm{II}],[\mathrm{S} \mathrm{II}]$, and [O III]. Among the three emission lines considered, $\sigma_{g}^{c}$ for [S II] formally correlates strongest with $\sigma_{*}$, and the ratio of the two has a somewhat smaller scatter. By comparison, [O III] exhibits the poorest performance. As Greene \& Ho (2005a) remarked, a possible explanation is that [O III], which has a significantly higher critical density than $[\mathrm{O} \mathrm{II}]$ and $[\mathrm{S} \mathrm{II}]$, originates from closer to the nucleus if the narrow-line region is density-stratified and hence has profile wings of higher velocity.

\footnotetext{
${ }^{6}$ As in our analysis, Greene \& Ho (2005a) decomposed [O III] using two Gaussians, one for the core and another for the wing; here we use their core component of [O III]. They only fit a single Gaussian for each of the doublet components of [O II] and [S II], but in view of the relative weakness of the wing component for the low-ionization lines in low-luminosity sources (see, e.g., Figure 9a), their single component is analogous to the core compoment of type 2 quasars.
} 
We verified that the above results are quite stable with respect to our particular choice of cut on data quality. Restricting the velocity dispersions to those with fractional errors $\leq 10 \%$ reduces the scatter of $\left\langle\sigma_{g}^{c} / \sigma_{*}\right\rangle$ from $\sim 0.35$ to $\sim 0.25$ for the type 2 quasar, at the expense of shrinking the sample, whereas relaxing the fractional errors to $30 \%$ results in a moderate increase of scatter on $\left\langle\sigma_{g}^{c} / \sigma_{*}\right\rangle$ but there is little effect on the significance of their statistical correlation. Our analysis shows that, as in lower-luminosity AGNs (e.g., Nelson \& Whittle 1996; Greene \& Ho 2005a; Ho 2009), the kinematics of the narrow-line region gas in type 2 quasars also approximately traces the virial motions of the stars in the central regions of their host galaxies. As such, the line widths of the narrow emission lines serve as an effective, economical substitute for the bulge stellar velocity dispersion, which is difficult, and at times impossible, to measure. Consistent with Greene \& Ho (2005a), we find that $\sigma_{g}$ only tracks $\sigma_{*}$ well when care is taken to remove the wings of the emission lines. Bian et al. (2006) reached a similar conclusion for a more limited number of type 2 quasars selected from the initial SDSS sample of Zakamska et al. (2003). For the three emission lines investigated here, the low-ionization line [S II] appears most promising. Its core velocity dispersion is, on average, essentially identical to $\sigma_{*}$, with a standard deviation of $32 \%$. The next best candidate is [O II], followed by [O III], as a last resort. Although the [O II] doublet is only marginally resolved by SDSS, and its $\sigma_{g}^{c}$ exhibits the largest deviation from $\sigma_{*}$, the deviation $(6 \%)$ is systematic and therefore correctable, and its scatter $(32 \%)$ is formally identical to that of [S II] (32\%). Still, priority should be given to [S II] whenever possible, as [O II] emanates not only from the narrow-line region but also from extra-nuclear emission-line regions. [O II] is prominent in H II regions (e.g., Gallagher et al. 1989) and traces galaxy-wide star formation, even in AGN hosts (Ho 2005).

\subsection{Nonvirial Motions: Dependence on AGN Properties}

The vast majority of type 2 quasars emit narrow-line gas with large, blueshifted velocities, not only in the highionization tracer [O III], but also in the low-ionization species [O II] and [S II]. This is most clearly seen in the large, systematic, positive offsets between $\sigma_{g}^{s}$ and $\sigma_{*}$ (Figure 9a). While the core component of the emission lines much better traces the gravitational potential of the stars (Figure $9 \mathrm{~b}$ ), a small fraction of our sample $(\sim 4 \%)$ still exhibits [O III] core velocity dispersions in excess of $400 \mathrm{~km} \mathrm{~s}^{-1}$, a couple as high as $\sim 1000 \mathrm{~km} \mathrm{~s}^{-1}$. Such velocities certainly do not reflect the virial motions of any realistic host galaxy. The central stellar velocity dispersions of nearby galaxies rarely exceed $350 \mathrm{~km} \mathrm{~s}^{-1}$ (Sheth et al. 2003). Instead, these large, super-virial velocities most likely arise from substantial energy injection into the narrow-line region, presumably from the AGN, which affect even the core velocities of the narrow emission lines. Moreover, while the core gas velocities are close, they are not exactly identical, to the stellar velocities. Do the residuals of the $\sigma_{g}-\sigma_{*}$ relation depend on AGN properties? Greene \& Ho (2005a; see also Bian et al. 2006) find that for [O III] the degree of offset between $\sigma_{*}$ and $\sigma_{g}^{s}$, which is sensitive to the wing component of the line, statistically scales with the Eddington ratio. The wing component is more blueshifted with increasing Eddington ratio. However, even the core of the line profile can be affected. Ho (2009) finds that while gravity is the primary agent responsible for the velocity broadening of the core of the [N II] line, the Eddington ratio acts as a secondary driver of the $\sigma_{g}^{c}-\sigma_{*}$ relation: sources with higher Eddington ratios have systematically larger core line widths relative to $\sigma_{*}$. Optical AGN luminosity and radio power also correlate with excess gaseous velocities, although Eddington ratio appears to be the more dominant factor (Ho 2009).

To evaluate whether these effects also apply to type 2 quasars, we need to quantify the $\mathrm{BH}$ mass and bolometric luminosity. In the absence of broad emission lines, the only viable method to estimate BH masses for type 2 sources is to make use of empirical scaling relations between $\mathrm{BH}$ masses and the properties of their host galaxies established for local samples of inactive galaxies (Kormendy \& Ho 2013). The most widely discussed correlations are those between BH mass and bulge stellar mass and stellar velocity dispersion. But challenges arise in connection with our intended current application to the SDSS-selected sample of type 2 quasars. First, the BH-bulge scaling relations (e.g., the $M_{\mathrm{BH}}-\sigma_{*}$ relation) has been shown to hold - with reasonable choices for the virial factor $f$ - mainly for nearby $(z \approx 0)$ type 1 (broad-line) AGNs (e.g., Onken et al. 2004; Ho \& Kim 2014). Our sample, by contrast, consists of type 2 AGNs at moderate redshift. In order to utilize the $M_{\mathrm{BH}}-\sigma_{*}$ relation to estimate their $\mathrm{BH}$ masses, we must assume (1) that the $M_{\mathrm{BH}}-\sigma_{*}$ relation applies to type 2 AGNs and (2) that we can neglect (or account for) its possible evolution with redshift, at least for the redshift range of our sample $(z \lesssim 0.8)$. Du et al. (2017) investigated the virial factors for a handful of Seyfert 2 galaxies with hidden broad-line regions and concluded that they are statistically consistent with the average virial factors of type 1 AGNs. This implies that both type 1 and type 2 AGNs have broad-line regions of similar structure and kinematics. In the present context, it suggests - at least tentatively - that the $M_{\mathrm{BH}}-\sigma_{*}$ relation can be applied to type 2 AGNs. The situation regarding the possible evolution of the $M_{\mathrm{BH}}-\sigma_{*}$ relation 
with redshift is unclear. On the one hand, a number of studies suggest that the zero point for the $M_{\mathrm{BH}}-\sigma_{*}$ relation increases toward higher redshifts, typically by $\sim 0.3-0.5$ dex when $z \approx 0.4-0.6$ (Woo et al. 2006, 2008; Treu et al. 2007). On the other hand, Shen et al. (2015) argue that this apparent evolution largely reflects selection effects, while Kormendy \& Ho (2013) show that the latest calibration of the local $M_{\mathrm{BH}}-\sigma_{*}$ relation essentially offsets the previously reported evolution of zero point at moderate redshifts. Thus, in the following analysis, we assume that our sample of low-redshift type 2 quasars obeys the local $M_{\mathrm{BH}}-\sigma_{*}$ relation. From Equation 5 of Kormendy \& Ho (2013),

$$
\log \left(\frac{M_{\mathrm{BH}}}{10^{9} M_{\odot}}\right)=-(0.500 \pm 0.049)+(4.429 \pm 0.295) \log \left(\frac{\sigma_{*}}{200 \mathrm{~km} \mathrm{~s}^{-1}}\right) .
$$

This relation has an intrinsic scatter of $0.28 \mathrm{dex}$. Note that the above relation applies only to classical bulges and elliptical galaxies, not to pseudobulges or to galaxy samples that contain a mixture of bulge types ${ }^{7}$. Apart from a minority of the objects (Zhao et al. 2018), we do not have reliable bulge classifications for most of our sample. In the subsequent analysis, we adopt Equation (2) to derive BH masses, but in Section 4.3 we will examine the consequences of relaxing this assumption.

The [O III] $\lambda 5007$ line offers the most practical means of tracing the level of AGN activity for the current sample of optically selected type 2 quasars. It is common practice to use the [O III] luminosity to estimate the AGN bolometric luminosity, but considerable debate surrounds the proper bolometric correction, the role of extinction correction, and whether or not the bolometric correction is luminosity-dependent (e.g., Heckman et al. 2004; Netzer et al. 2006; Kauffmann \& Heckman 2009; Lamastra et al. 2009; Stern \& Laor 2012; Pennell et al. 2017; see discussion in Heckman \& Best 2014). For concreteness, we adopt a constant bolometric correction of 600 (i.e., $L_{\text {bol }}=600 L_{[\mathrm{O} \text { III] }}$ ) to the extinction-corrected [O III] luminosity, following the recommendation of Kauffmann \& Heckman (2009), with an uncertainty of \pm 150 . For comparison, we will also consider the constant bolometric correction of 3500 for extinctionuncorrected [O III] luminosity originally advocated by Heckman et al. (2004), as well as the luminosity-dependent, extinction-corrected bolometric corrections of Lamastra et al. (2009), as parameterized by Trump et al. (2015). We estimate the internal extinction from the observed Balmer decrement, assuming an intrinsic value of $\mathrm{H} \alpha / \mathrm{H} \beta=3.1$ appropriate for AGNs (Halpern \& Steiner 1983) and the extinction curve of Cardelli et al. (1989). For objects for which the Balmer decrement could not be measured, we adopt the median value of $\mathrm{H} \alpha / \mathrm{H} \beta=4.04$ obtained from the objects for which this quantity was measured.

Figure 10a shows the relation between $\Delta \sigma^{s} \equiv \log \sigma_{g}^{s}-\log \sigma_{*}$ and $L_{\mathrm{bol}} / L_{\mathrm{Edd}}$, both for our sample of type 2 quasars (red points) and for lower luminosity sample of Seyferts from Greene \& Ho (2005a; small grey points). We consistently recalculate the black hole masses and bolometric luminosities of the Greene \& Ho objects using the $M_{\mathrm{BH}}-\sigma_{*}$ relation adopted in this work (Equation 2) and the bolometric correction of 600 for extinction-corrected [O III] luminosity ${ }^{8}$. The velocity residuals of all three transitions correlate positively and strongly with the Eddington ratio, both for the high-luminosity and low-luminosity samples. The combination of the lower luminosity Greene \& Ho sources and our high-luminosity quasars provides the largest dynamic range in Eddington ratio, yielding the following functional dependence between $\Delta \sigma^{s}$ and $L_{\mathrm{bol}} / L_{\mathrm{Edd}}$ (black solid line in Figure 10a):

$$
\Delta \sigma^{s}=\left\{\begin{array}{l}
(0.106 \pm 0.004) \log \left(L_{\mathrm{bol}} / L_{\mathrm{Edd}}\right)+(0.255 \pm 0.009) \text { for }[\mathrm{O} \mathrm{II}] \\
(0.093 \pm 0.004) \log \left(L_{\mathrm{bol}} / L_{\mathrm{Edd}}\right)+(0.222 \pm 0.009) \text { for }[\mathrm{S} \mathrm{II}] \\
(0.149 \pm 0.005) \log \left(L_{\mathrm{bol}} / L_{\mathrm{Edd}}\right)+(0.392 \pm 0.010) \text { for }[\mathrm{O} \mathrm{III}]
\end{array}\right.
$$

with Spearman's rank correlation coefficients of $\rho=0.43,0.38$, and 0.56 , respectively, and $P_{\text {null }}<10^{-6}$. Greene \& Ho (2005a) already presented the results for [O III] for type 2 Seyferts. Here we confirm that it extends to sources of even higher luminosity and Eddington ratio, and, moreover, that it also applies to the low-ionization lines [O II] and [S II].

More surprisingly, we find that even the core component of the lines are affected systematicaly by Eddington ratio (Figure 10b). The type 2 quasars exhibit the strongest correlation between $\Delta \sigma^{c}$ and $L_{\mathrm{bol}} / L_{\mathrm{Edd}}(\rho=0.66,0.49$, and 0.61 for $[\mathrm{O} \mathrm{II}]$, [S II], and $\left.[\mathrm{O} \mathrm{III}] ; P_{\text {null }}<10^{-6}\right)$, but statistically significant correlations also exist for the lower-luminosity objects of Greene \& Ho (2005a) alone $\left(\rho=0.36,0.32\right.$, and 0.14 for $[\mathrm{O} \mathrm{II}]$, [S II], and [O III]; $\left.P_{\text {null }}<10^{-6}\right)$, and for

\footnotetext{
${ }^{7}$ She et al. (2017) provide a fit for the $M_{\mathrm{BH}}-\sigma_{*}$ relation of all galaxies with reliable BH masses from Kormendy \& Ho (2013), regardless of bulge type: $\log \left(M_{\mathrm{BH}} / 10^{9} M_{\odot}\right)=(-0.68 \pm 0.05)+(5.20 \pm 0.37) \log \left(\sigma / 200 \mathrm{~km} \mathrm{~s}^{-1}\right)$, with an intrinsic scatter of 0.44 dex.

8 The [O III] luminosities of Greene \& Ho (2005a) were not corrected for internal extinction. For consistency with the conventions of our study, we applied an internal extinction correction to the luminosities of Greene \& Ho (2005a) using Balmer decrements for their objects obtained from the JHU/MPA database (https://wwwmpa.mpa-garching.mpg.de/SDSS)
} 
both samples combined $\left(\rho=0.33,0.26\right.$, and 0.13 for $[\mathrm{O} \mathrm{II}]$, [S II], and [O III]; $\left.P_{\text {null }}<10^{-6}\right)$. The sensitivity of $\sigma_{g}^{c}$ to $L_{\mathrm{bol}} / L_{\mathrm{Edd}}$ echoes the findings of Ho (2009; overplotted as dotted line in the [O II] and [S II] panels of Figure 10b) based on the low-ionization line $[\mathrm{N} \mathrm{II}] \lambda 6583$, which extend the Eddington ratios down by $\sim 2$ orders of magnitude, to $L_{\mathrm{bol}} / L_{\mathrm{Edd}} \approx 10^{-5.5}$.

We looked for, but failed to find, any dependence between velocity excess (either $\Delta \sigma^{s}$ or $\Delta \sigma^{c}$ ) and [O III] luminosity or $20 \mathrm{~cm}$ radio power (available from the FIRST database; Becker et al. 1995). Greene \& Ho (2005a) arrived at a similar conclusion for $\Delta \sigma^{s}$. The mildly significant correlation between $\Delta \sigma^{c}([\mathrm{~N} \mathrm{III}])$ and $\mathrm{H} \alpha$ luminosity and radio power seen by Ho (2009), which he considers to be secondary to the influence of Eddington ratio, may be masked by the larger measurement uncertainties of the present data.

\subsection{Black Hole Masses and Eddington Ratios}

One of the main objectives of this study is to evaluate the BH masses and Eddington ratios of type 2 quasars. We estimate $\mathrm{BH}$ masses using the $M_{\mathrm{BH}}-\sigma_{*}$ relation (Equation 2). Having established that the kinematics of the narrow-line region in type 2 quasars largely tracks the virial velocities of the bulge, we use the velocity dispersion of the core component $\left(\sigma_{g}^{c}\right)$ of the narrow lines to estimate $\sigma_{*}$ whenever the latter is unavailable. We give preference to $\sigma_{g}^{c}$ from [S II], followed by [O II], and then [O III]. We adjust the gas velocity dispersions by applying a small statistical zeropoint scaling correction: $\left\langle\sigma_{g}^{c} / \sigma_{*}\right\rangle=0.99,1.06$, and 1.00 for [S II], [O II], and [O III]. As mentioned in Section 4.2, a small fraction of the objects have exceptionally large [O III] line widths of clearly non-gravitational origin. We omit these from the following analysis.

The $\mathrm{BH}$ masses of type 2 quasars range from $M_{\mathrm{BH}} \approx 10^{6.5}$ to $10^{10.4} M_{\odot}$, with a median value of $10^{8.2} M_{\odot}$ (Figure 11a). Had we adopted the $M_{\mathrm{BH}}-\sigma_{*}$ relation of She et al. (2017; see footnote 7), which does not distinguish between classical and pseudo bulges, the median BH mass would decrease by $\sim 0.25$ dex. The Eddington ratios span $L_{\mathrm{bol}} / L_{\mathrm{Edd}} \approx 10^{-2.9}$ to $10^{1.8}$, with a median value of $10^{-0.7}$ (Figure $11 \mathrm{~b}$ ). These $\mathrm{BH}$ masses and Eddington ratios are very similar to the results of Greene et al. (2009). The alternative [O III] bolometric corrections of Trump et al. (2015) yield a lower median $L_{\text {bol }} / L_{\text {Edd }}$ lower by $\sim 0.2$ dex, while Heckman et al.'s (2004) bolometric correction would lead to an increase of $\sim 0.4$ dex in the median $L_{\mathrm{bol}} / L_{\mathrm{Edd}}$. Figure 12 compares type 2 quasars with optically selected type 1 AGNs over the same redshift range $(z<0.83)$ studied by Shen et al. (2011). Roughly half of the type 1 sources have $L_{[\mathrm{O} \text { III] }]} \gtrsim 2 \times 10^{42}$ $\mathrm{erg} \mathrm{s}^{-1}$, the lower limit threshold adopted for type 2 quasars (Reyes et al. 2008). The BH masses for the type 1 sources, taken directly from Shen et al. (2011), are based on single-epoch virial mass estimators using the broad $\mathrm{H} \beta$ or $\mathrm{Mg}$ II emission lines. For consistency with the type 2 sources, we also compute the bolometric luminosities of the type 1 sample using $L_{[\mathrm{O} \text { III }]}$ from Shen et al., but in this instance we need to use the [O III] bolometric correction of Heckman et al. (2004) because extinction corrections are not available. Taken at face value, type 2 quasars cover a similar range of $\mathrm{BH}$ masses and Eddington ratios as type 1 quasars, with type 1 systems tending toward somewhat higher $M_{\mathrm{BH}}\left(\right.$ median $\left.10^{8.5} M_{\odot}\right)$ and lower $L_{\mathrm{bol}} / L_{\mathrm{Edd}}\left(\operatorname{median} 10^{-0.9}\right)$. The lower-luminosity type 2 Seyferts of Greene \& Ho (2005a) differ more dramatically from type 2 quasars (median $M_{\mathrm{BH}}=10^{7.9} M_{\odot}$; median $L_{\mathrm{bol}} / L_{\mathrm{Edd}}=10^{-2.1}$ ), but this is a trivial consequence of sample selection (the optically selected type 2 quasars are required to meet the luminosity threshold of quasars).

Significant uncertainties and selection effects complicate the comparison between type 1 and type 2 quasars (e.g., $\mathrm{BH}$ mass estimation, bolometric corrections, dust extinction, etc), and we do not recommend taking too literally the numerical values for any individual object or of the apparent minor differences between the two quasar populations. Nevertheless, it is worth remarking that the bolometric luminosities for $\sim 20 \%$ of the type 2 quasars formally exceed the Eddington limit, some by a significant margin; 11 objects $(1.6 \%)$ have $L_{\text {bol }} / L_{\text {Edd }} \geq 10$. The sources with the most extreme Eddington ratios have the highest luminosities $\left(L_{[\mathrm{O} \text { III] }} \gtrsim 10^{43.5} \mathrm{erg} \mathrm{s}^{-1}\right.$ and lowest $\mathrm{BH}$ masses $\left(M_{\mathrm{BH}} \approx 10^{6.5}-10^{7.5} M_{\odot}\right)$. Type 2 quasars, as a population, appear to have somewhat higher accretion rates than their type 1 counterparts, and the subset with the least massive BHs are radiating at highly super-Eddington rates. This may arise naturally in the evolutionary scenario linking obscured and unobscured AGNs.

\section{SUMMARY}

We analyze the optical spectra of the 887 low-redshift $(0.037<z<0.83)$ type 2 quasars from the SDSS catalog of Reyes et al. (2008), with the aim of deriving two fundamental physical parameters for this population, their BH mass and Eddington ratio. Our strategy is to estimate BH masses using the $M_{\mathrm{BH}}-\sigma_{*}$ relation, employing directly measured central stellar velocity dispersions whenever possible and otherwise indirectly via the velocity dispersions of the bright narrow lines [O II], [S II], and [O III]. 
Our main results are as follows:

1. The kinematics of the core component of the narrow emission lines trace the gravitational potential of the stars of the host galaxy, albeit with significant scatter. The low-ionization lines [S II] and [O II] are most effective, but even the high-ionization line [O III] can be used.

2. The AGN plays a secondary but still important role in governing the kinematics of narrow-line region. Narrow emission lines of both low and high ionization exhibit significantly blueshifted velocity wings. The magnitude of these nonvirial motions becomes more prominent with increasing Eddington ratio.

3. Optically selected type 2 quasars have $\mathrm{BH}$ masses spanning $M_{\mathrm{BH}} \approx 10^{6.5}$ to $10^{10.4} M_{\odot}\left(\right.$ median $\left.10^{8.2} M_{\odot}\right)$ and Eddington ratios $L_{\mathrm{bol}} / L_{\mathrm{Edd}} \approx 10^{-2.9}$ to $10^{1.8}$ (median $10^{-0.7}$ ). A minosity exceeds the Eddington limit by more than a factor of 10 .

4. The accretion rates of type 2 quasars are somewhat higher than those of type 1 quasars of similar redshift.

This work was supported by the National Key R\&D Program of China (2016YFA0400702), the National Science Foundation of China (11473002, 11721303), the National Youth Fund (11303008), and the Youth Foundation of Hebei Province of China (A2011205067). We thank Jenny Greene for sending the data from Greene \& Ho (2005a). MK is grateful to Jian-Min Wang for his hospitality and help during her visit to IHEP and to JinLin Han for his help during her stay at NAOC. She thanks Song Huang, Ligang Hou, Xianmin Meng, Jinyi Shangguan, Jiayi Sun, and Junzhi Wang for their help.

Funding for the SDSS has been provided by the Alfred P. Sloan Foundation, the Participating Institutions, the National Science Foundation, the U.S. Department of Energy, the National Aeronautics and Space Administration, the Japanese Monbukagakusho, the Max Planck Society, and the Higher Education Funding Council for England. The SDSS Web Site is http://www.sdss.org/

The SDSS is managed by the Astrophysical Research Consortium for the Participating Institutions. The Participating Institutions are the American Museum of Natural History, Astrophysical Institute Potsdam, University of Basel, University of Cambridge, Case Western Reserve University, University of Chicago, Drexel University, Fermilab, the Institute for Advanced Study, the Japan Participation Group, Johns Hopkins University, the Joint Institute for Nuclear Astrophysics, the Kavli Institute for Particle Astrophysics and Cosmology, the Korean Scientist Group, the Chinese Academy of Sciences (LAMOST), Los Alamos National Laboratory, the Max-Planck-Institute for Astronomy (MPIA), the Max-Planck-Institute for Astrophysics (MPA), New Mexico State University, Ohio State University, University of Pittsburgh, University of Portsmouth, Princeton University, the United States Naval Observatory, and the University of Washington.

\section{REFERENCES}

Abazajian, K., Adelman-McCarthy, J. K., Agüeros, M. A., et al. 2009, ApJS, 182, 543

Alexandroff, R., Strauss, M. A., Greene, J. E., et al. 2013, MNRAS, 435, 3306

Antonucci, R. 1993, ARA\&A, 31, 473

Ballantyne, D. R. 2016, MNRAS, 464, 626

Barth, A. J., Ho, L. C., \& Sargent, W. L. W. 2002, AJ, 124, 2607

Becker, R. H., White, R. L., \& Helfand, D. J. 1995, ApJ, 450,559

Beifiori, A., Maraston, C., Thomas, D. \& Johansson, J. 2011, A\&A, 531, A109

Bender, R. 1990, A\&A, 229, 441
Bessiere, P., Tadhunter, C. N., Ramos Almeida, C., \& Villar-Martin, M. 2012, MNRAS, 426, 276

Bianchi, S., Marinucci, A., Matt, G., et al. 2017, MNRAS, 468,2740

Bruzual, G., \& Charlot, S. 2003, MNRAS, 344, 1000

Cappellari, M., \& Emsellem, E. 2004, PASP, 116,138

Cardelli, J. A., Clayton, G. C., \& Mathis, J. S., 1989, ApJ, 345,245

Dressler, A. 1984, ApJ, 286, 97

Du, P., Wang, J.-M., \& Zhang, Z.-X. 2017, ApJ, 840, L6

Elitzur, M., \& Ho, L. C. 2009, ApJ, 701, L91

Elitzur, M., Ho, L. C., \& Trump, J. R. 2014, MNRAS, 438, 3340

Elitzur, M., \& Netzer, H. 2016, MNRAS, 459, 585 
Ferrarese, L., \& Merritt, D. 2000, ApJ, 539, L9

Filippenko, A. V., \& Halpern, J. P. 1984, ApJ, 285, 459

Gallagher, J. S., Bushouse, H., \& Hunter, D. A. 1989, AJ, 97,700

Ge, J.-Q., Hu, C., Wang, J.-M., Bai, J.-M., \& Zhang, S. 2012, ApJS, 201, 32

Gebhardt, K., Bender, R., Bower, G., et al. 2000, ApJ, 539, L13

Gelderman, R., \& Whittle, M. 1994, ApJS, 91, 491

Greene, J. E., \& Ho, L. C. 2005a, ApJ, 627, 721

Greene, J. E., \& Ho, L. C. 2005b, ApJ, 630, 122

Greene, J. E., \& Ho, L. C. 2006, ApJ, 641, 117 (GH06)

Greene, J. E. , Zakamska, N. L., Liu, X., et al. 2009, ApJ, 702,441

Halpern, J. P., \& Steiner, J. E. 1983, ApJ, 269, L37

Häring, N., \& Rix, H.-W. 2004, ApJ, 604, L89

Heckman, T. M., \& Best, P. N. 2014, ARA\&A, 52, 589

Heckman, T., Kauffmann, G., Brinchmann, J., et al. 2004, ApJ, 613, 109

Ho, L. C. 2005, ApJ, 629, 680

Ho, L. C. 2008, ARA\&A, 46, 475

Ho, L. C. 2009, ApJ, 699, 638

Ho, L. C., Filippenko, A. V., \& Sargent, W. L. W. 1996, ApJ, 462, 183

Ho, L. C., Filippenko, A. V., Sargent, W. L. W. 1997a, ApJS, 112, 315

Ho, L. C., Filippenko, A. V., Sargent, W. L. W., \& Peng, C. Y. 1997b, ApJS, 112, 391

Ho, L. C., Greene, J. E., Filippenko, A. V., \& Sargent, W.

L. W. 2009, ApJS, 183, 1

Ho, L. C., \& Kim, M. 2014, ApJ, 789, 17

Ho, L. C., \& Kim, M. 2015, ApJ, 809, 123

Ho, L. C., Kim, M., \& Terashima, Y. 2012, ApJ, 759, L16

Hopkins, P. F., Hernquist, L., Cox, T. J., et al. 2006, ApJS, 163, 1

Kaspi S., Smith P. S., Netzer H., et al. 2000, ApJ, 533, 631

Kauffmann, G., \& Heckman, T. 2009, MNRAS, 397, 135

Kelson, D. D., Illingworth, G. D., van Dokkum, P. G., \&

Franx, M. 2000, ApJ, 531, 159

Kim, M., Ho, L. C., \& Im, M. 2006, ApJ, 642, 702

Kormendy, J., \& Ho, L. C. 2013, ARA\&A, 51, 511

Lal, D. V., \& Ho, L. C. 2010, AJ, 139, 1089

Lamastra, A., Bianchi, S., Matt, G., et al. 2009, A\&A, 504, 73

Liu, X., Shen, Y., Strauss, M. A., \& Greene, J. E. 2010, ApJ, 708, 427

Liu, X., Zakamska, N. L., Greene, J. E., et al. 2009, ApJ, 702, 1098

Miniutti, G., Saxton, R. D., Rodriguez-Pascual, P. M., et al. 2013, MNRAS, 433, 1764
Nelson, C. H. 2000, ApJ, 544, L91

Nelson, C. H., \& Whittle, M. 1996, ApJ, 465, 96

Netzer, H. 2015, ARA\&A, 53, 365

Netzer, H., Mainier, V., Rosati, P., \& Trakhtenbrot, B. 2006, A\&A, 453, 525

Onken, C. A., Ferrarese, L., Merritt, D., et al. 2004, ApJ, 615,645

Osterbrock, D. E. 1989, Astrophysics of Gaseous Nebulae and Active Galactic Nuclei (Mill Valley: Univ. Science Books)

Pennell, A., Runnoe, J. C., \& Brotherton, M. 2017, MNRAS, 468, 1433

Reyes, R., Zakamska, N. L., Strauss, M. A., et al. 2008, AJ, 136,2373

Sánchez-Blázquez, P., Peletier, R. F., Jiménez-Vicente, J., et al. 2006, MNRAS, 371, 703

Sanders, D. B., Soifer, B. T., Elias, J. H., et al. 1988, ApJ, 325,74

Sargent, W. L. W., Schechter, P. L., Boksenberg, A., \& Shortridge, K. 1977, ApJ, 212, 326

Schlafly, E. F., \& Finkbeiner, D. P. 2011, ApJ, 737, 103

Schmidt, M., \& Green, R. F. 1983, ApJ, 269, 352

She, R., Ho, L. C., \& Feng, H. 2017, ApJ, 835, 223

Shen, Y., Greene, J. E., Ho, L. C., et al. 2015, ApJ, 805, 96

Shen, Y., Richards, G. T., Strauss, M. A., et al. 2011, ApJS, 194, 45

Sheth, R. K., Bernardi, M., Schechter, P. L., et al. 2003, ApJ, 594, 225

Shu, X. W., Wang, T. G., Jiang, N., et al. 2017, ApJ, 837, 3 Stern, J., \& Laor, A. 2012, MNRAS, 426, 2703

Tonry, J., \& Davis, M. 1979, AJ, 84, 1511

Tremaine, S., Gebhardt, K., Bender, R., et al. 2002, ApJ, 574,740

Treu, T., Woo, J.-H., Malkan, M. A., \& Blandford, R. D. 2007, ApJ, 667, 117

Trump, J. R., Sun, M., Zeimann, G. R., et al. 2015, ApJ, 811,26

Urry, C. M., \& Padovani, P. 1995, PASP, 107, 803

Valdes, F., Gupta, R., Rose, J. A., Singh, H. P., \& Bell, D. J. 2004, ApJS, 152, 251

Veilleux, S. 1991, ApJS, 75, 383

Vestergaard, M., \& Peterson, B. M. 2006, ApJ, 641, 689

Whittle, M. 1985, MNRAS, 213, 1

Whittle, M. 1992, ApJ, 387, 109

Whittle, M., Pedlar, A., Meurs, E. J. A., et al. 1988, ApJ, 326,125

Woo, J.-H., Treu, T., Malkan, M. A., \& Blandford, R. D. 2006, ApJ, 645, 900

Woo, J.-H., Treu, T., Malkan, M. A., \& Blandford, R. D. 2008, ApJ, 681, 925 
Zakamska, N. L., Gomez, L., Strauss, M. A., \& Krolik, J. H. 2008, AJ, 136, 1607

Zakamska, N. L., Lampayan, K., Petric, A., et al. 2016, MNRAS, 455, 4191
Zakamska, N. L., Strauss, M. A., Krolik, J. H., et al. 2003, AJ, 126, 2125

Zhao, D., Ho, L. C., Zhao, Y.-Li., Kim, M., \& Shangguan, J. 2018, in preparation 
Table 1. Sample Properties

\begin{tabular}{|c|c|c|c|c|c|c|c|c|c|c|c|c|c|c|c|}
\hline $\begin{array}{l}\text { ject Name } \\
\text { SDSS } \\
(1)\end{array}$ & (2) & $\begin{array}{c}A_{V} \\
\text { (mag) } \\
(3)\end{array}$ & (4) & $\begin{array}{l}\mathrm{EW} \\
(\AA) \\
(5)\end{array}$ & (6) & $\begin{array}{c}\left.L_{[\mathrm{O}} \mathrm{III}\right] \\
\left(\mathrm{erg} \mathrm{s}^{-1}\right) \\
(7)\end{array}$ & $\begin{array}{c}\sigma_{*} \\
\left(\mathrm{~km} \mathrm{~s}^{-1}\right) \\
(8)\end{array}$ & $\begin{array}{c}\left.\sigma_{[\mathrm{S}}^{c} \mathrm{II}\right] \\
\left(\mathrm{km} \mathrm{s}^{-1}\right) \\
(9)\end{array}$ & $\begin{array}{c}\left.\sigma_{[\mathrm{O}}^{c} \mathrm{II}\right] \\
\left(\mathrm{km} \mathrm{s}^{-1}\right) \\
(10)\end{array}$ & $\begin{array}{c}\left.\sigma_{[\mathrm{O}}^{c} \mathrm{III}\right] \\
\left(\mathrm{km} \mathrm{s}^{-1}\right) \\
(11)\end{array}$ & $\begin{array}{c}\left.\sigma_{[\mathrm{S}}^{\mathrm{s}} \mathrm{II}\right] \\
\left(\mathrm{km} \mathrm{s}^{-1}\right) \\
(12)\end{array}$ & $\begin{array}{c}\left.\sigma_{[\mathrm{O}}^{\mathrm{s}} \mathrm{II}\right] \\
\left(\mathrm{km} \mathrm{s}^{-1}\right) \\
(13)\end{array}$ & $\begin{array}{c}\left.\sigma_{[\mathrm{O}}^{\mathrm{s}} \mathrm{III}\right] \\
\left(\mathrm{km} \mathrm{s}^{-1}\right) \\
(14)\end{array}$ & $\begin{array}{c}M_{\mathrm{BH}} \\
\left(M_{\odot}\right) \\
(15)\end{array}$ & $\mathrm{bol} / L_{\mathrm{Edd}}$ \\
\hline $0259.10+$ & 007 & 762 & 1 & 5 & & .05 & 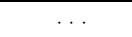 & . & $\cdots$ & 30.0 & . & 5.3 & 8.6 & & 30 \\
\hline J000351.83-0 & 2689 & 0968 & 1 & 0. & 4.04 & $.19 \pm 0.05$ & $241.7 \pm 65.5$ & . & $\cdots$ & $25.4 \pm$ & . & 7.7 & $76.9=$ & . & 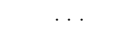 \\
\hline J000453.43-005038.4 & 0.6430 & 0.1100 & 3.1 & $<3$ & 4.04 & $43.31 \pm 0.05$ & $\cdots$ & $\cdots$ & $137.3 \pm 9.8$ & $174.0 \pm 2.0$ & $\cdots$ & $382.6 \pm 15.8$ & $500.5 \pm 42.0$ & $7.66 \pm 0.75$ & $0.33 \pm 0.76$ \\
\hline $\mathrm{J} 000728.83+010604.0^{a}$ & 0.4663 & 0.0988 & 7.8 & 6.0 & 4.04 & $42.37 \pm 0.05$ & $203.4 \pm 50.6$ & $\cdots$ & $\cdots$ & $\cdots$ & $\cdots$ & $599.3 \pm 104.7$ & $402.0 \pm 93.1$ & $\cdots$ & $\cdots$ \\
\hline J001000.75-011008.5 & 0.5823 & 0.0936 & 6.1 & $<3$ & 4.04 & $42.71 \pm 0.06$ & $\cdots$ & & $.6 \pm 57.1$ & $204.4 \pm 5$ & $\cdots$ & $.4 \pm 41.9$ & $260.1 \pm 13.8$ & $9 \pm 0.79$ & $-2.30 \pm$ \\
\hline J001111.95+005626.3 & 0.4094 & 0.0812 & 6.9 & $<3$ & 4.04 & $42.69 \pm 0.05$ & $\cdots$ & $\cdots$ & $133.6 \pm 17.5$ & $138.7 \pm 31.6$ & $\cdots$ & $414.3 \pm 43.0$ & $289.6 \pm 19.4$ & $7.61 \pm 0.78$ & $-0.25 \pm 0.79$ \\
\hline J001126.95+155329.6 & 0.0999 & 0.2062 & 12.3 & 5.5 & $3.76 \pm 0.05$ & $42.14 \pm 0.02$ & $154.9 \pm 19.4$ & $141.2 \pm 5.9$ & $159.1 \pm 8.2$ & $134.9 \pm 5.5$ & $247.6 \pm 31.3$ & $225.5 \pm 18.2$ & $224.0 \pm 6.7$ & $8.01 \pm 0.37$ & $-1.19 \pm 0.39$ \\
\hline J001206.31-094725.6 & 0.1668 & 0.1057 & 13.8 & 5.2 & $5.17 \pm 0.22$ & $42.92 \pm 0.06$ & $146.7 \pm 21.2$ & $114.0 \pm 7.4$ & $167.5 \pm 43.9$ & $154.0 \pm 7.4$ & $242.0 \pm 11.5$ & $423.1 \pm 34.3$ & $285.0 \pm 18.4$ & $7.90 \pm 0.40$ & $-0.30 \pm 0.42$ \\
\hline J002016.88-093244.7 & 0.3600 & 0.1289 & 20.9 & $<3$ & $5.17 \pm 0.31$ & $43.06 \pm 0.08$ & $\ldots$ & $\ldots$ & $182.9 \pm 8.5$ & $176.0 \pm 4.2$ & $\ldots$ & $376.3 \pm 31.7$ & $469.9 \pm 4.2$ & $8.22 \pm 0.74$ & $-0.48 \pm 0.75$ \\
\hline
\end{tabular}

Note-

${ }^{a}$ Kinematically complex emission-line core.

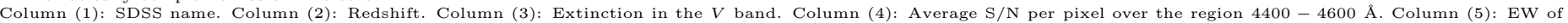

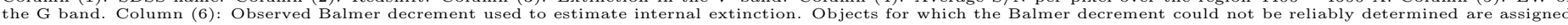

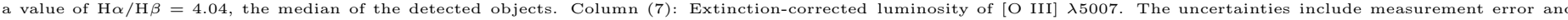

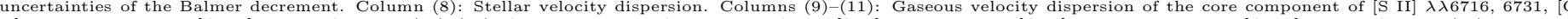

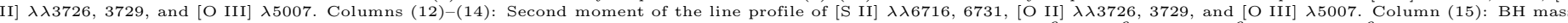

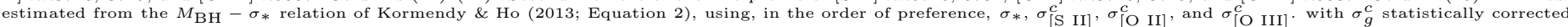

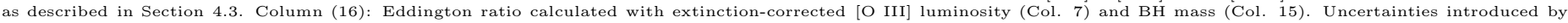

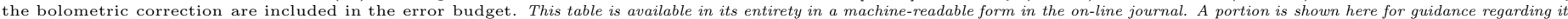
form and content. 


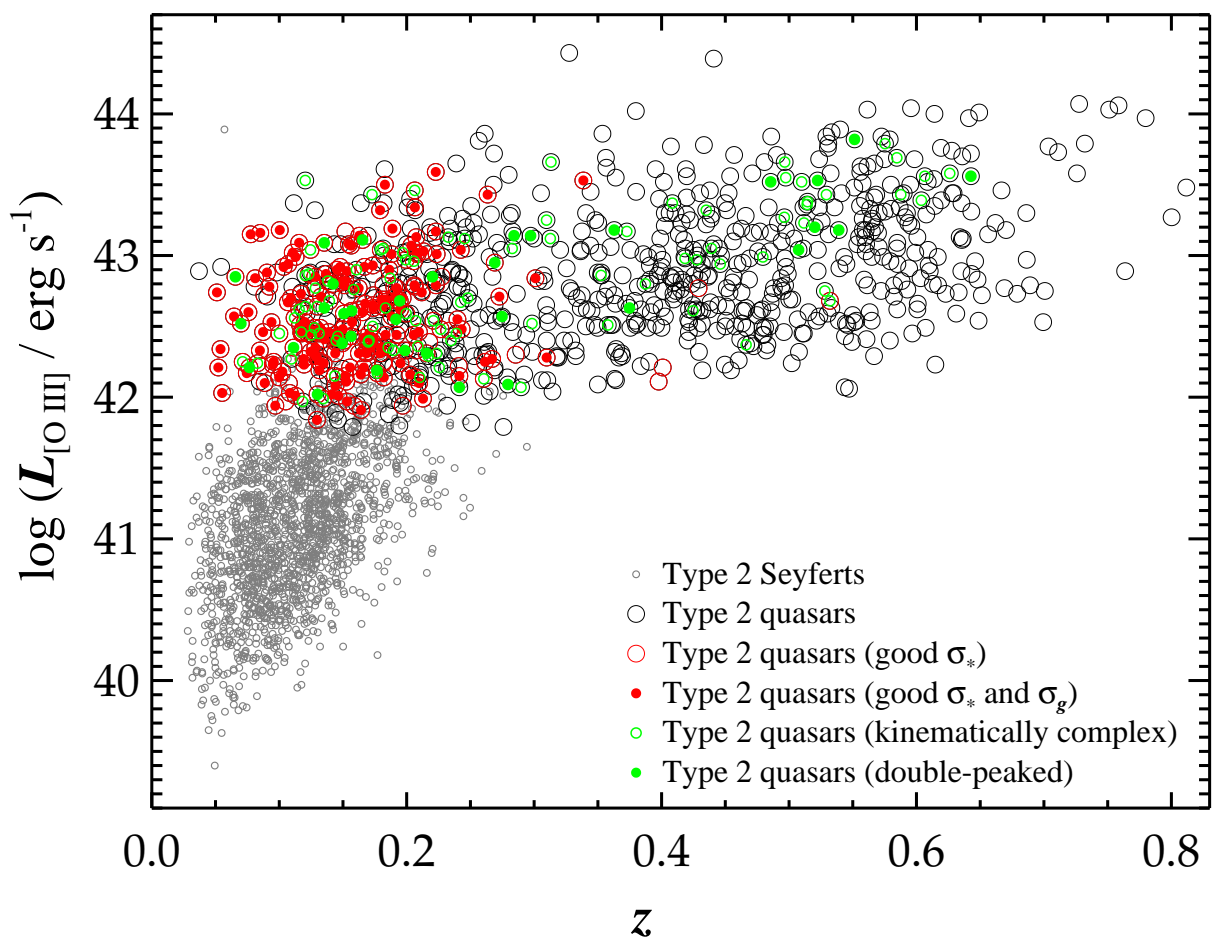

Figure 1. Distribution of [O III] luminosities and redshifts of our parent sample of 887 type 2 quasars (open black symbols). Plotted in red are the 219 objects that have $\sigma_{*}$ measurements with fractional errors $\leq 20 \%$, with the final sample of 142 objects having robust values of both $\sigma_{*}$ and $\sigma_{g}$ further highlighted as filled red symbols. Green symbols denote objects with kinematically complex emission-line cores, and green filled symbols mark the subset with double-peaked profiles. The small grey points are the lower luminosity type 2 AGNs (Seyferts) from Greene \& Ho (2005a). 


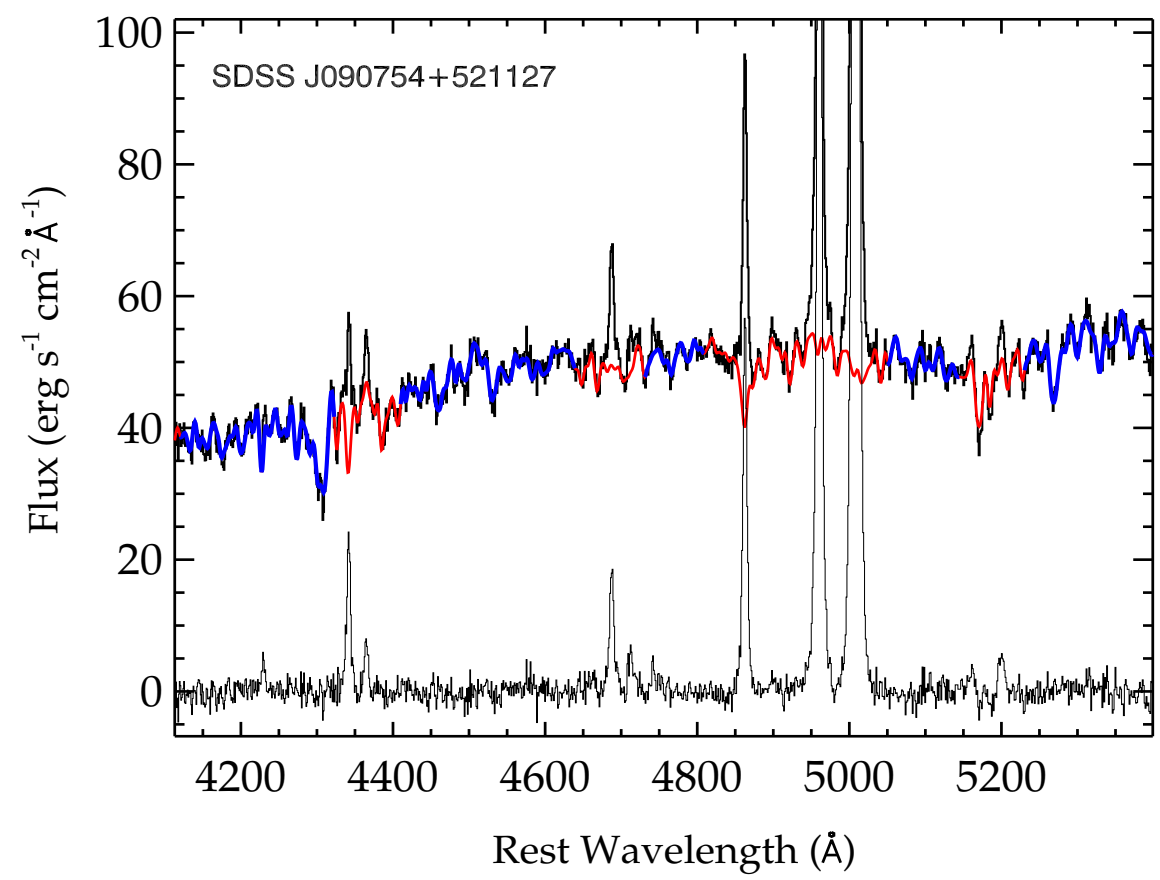

Figure 2. Illustration of the method for $\sigma_{*}$ measurement. The SDSS spectrum, corrected for extinction and redshift, is plotted as thick black histograms. The best-fit model, a scaled, broadened composite stellar template comprising several individual stars (F, K, and M late-type red giants), is plotted in blue. Regions of the spectrum containing strong emissions are excluded from the fit (red). The residuals (data minus best-fit model) are plotted on the bottom.

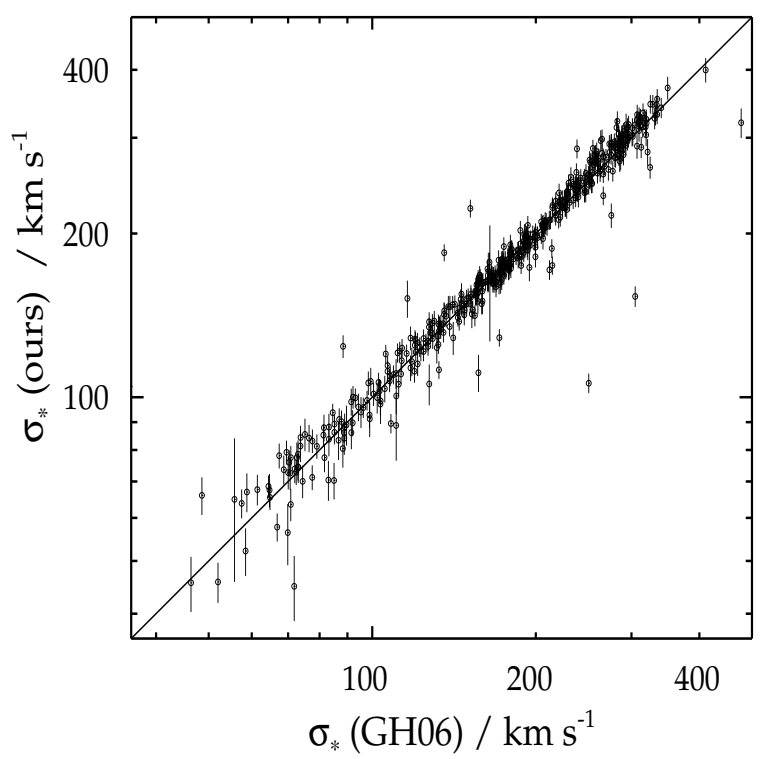

Figure 3. Comparison between our measurements of $\sigma_{*}$ and those of GH06 for their reference sample of 504 active galaxies. The agreement is excellent. The mean difference between the two sets of measurements is $\left\langle\Delta \sigma_{*}\right\rangle=0.002 \pm 0.05$, where $\Delta \sigma_{*} \equiv \log \sigma_{*}($ ours $)-$ $\log \sigma_{*}(\mathrm{GH} 06)$. 

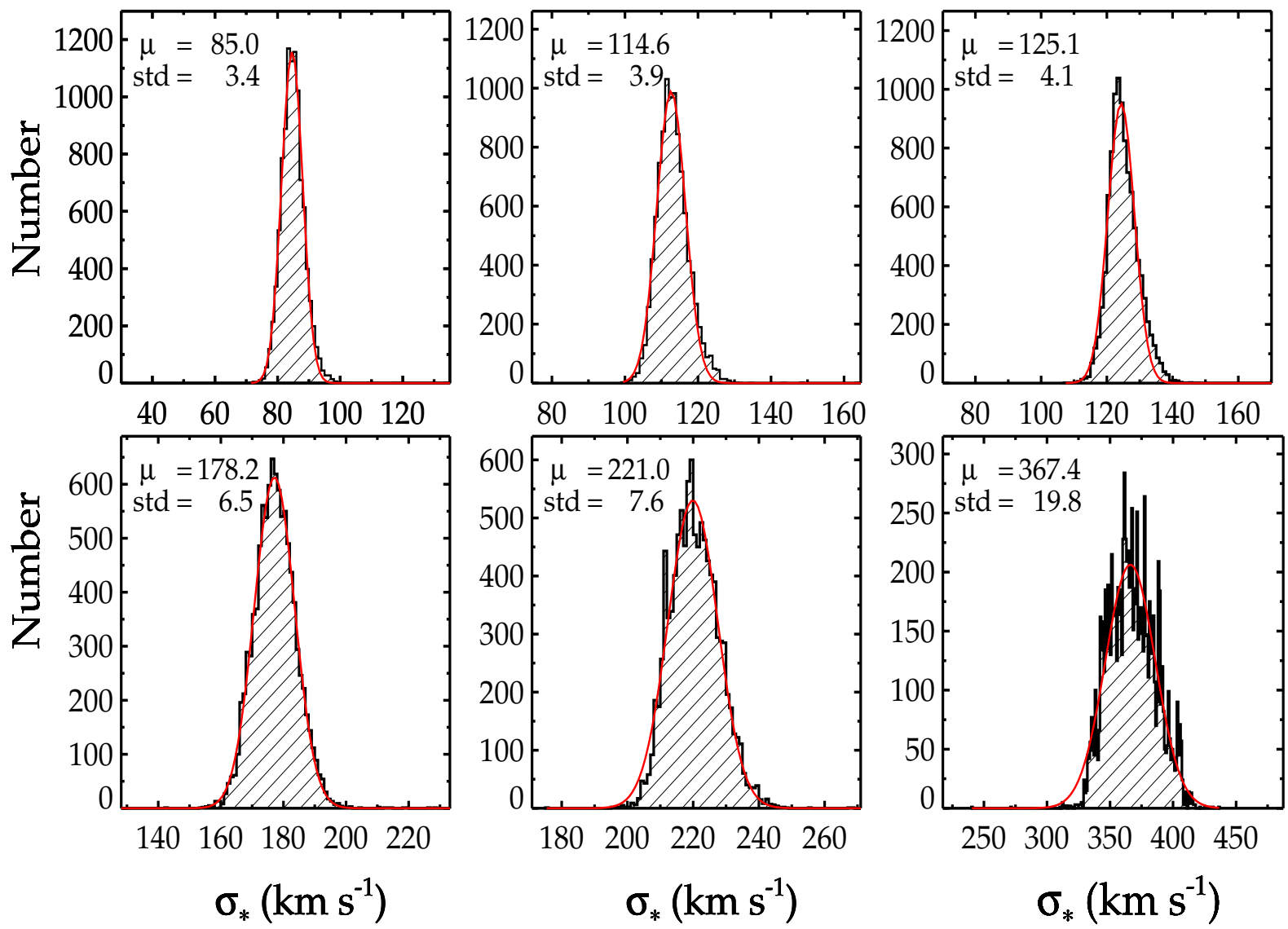

Figure 4. The effect of template mismatch on measured velocity dispersions. For each of six high-S/N ( $\gtrsim 90)$ SDSS galaxy spectra with $\sigma_{*}=85,114,125,178,220$, and $360 \mathrm{~km} \mathrm{~s}^{-1}$, we perform $10^{4}$ fits with different stellar templates constructed from random combinations of stars with spectral type A, F, G, K, and M. The mean $(\mu)$ of the resulting distribution of $\sigma_{*}$ and its standard deviation from a Gaussian fit (red line) to the distribution are given in the top left corner of each panel. 

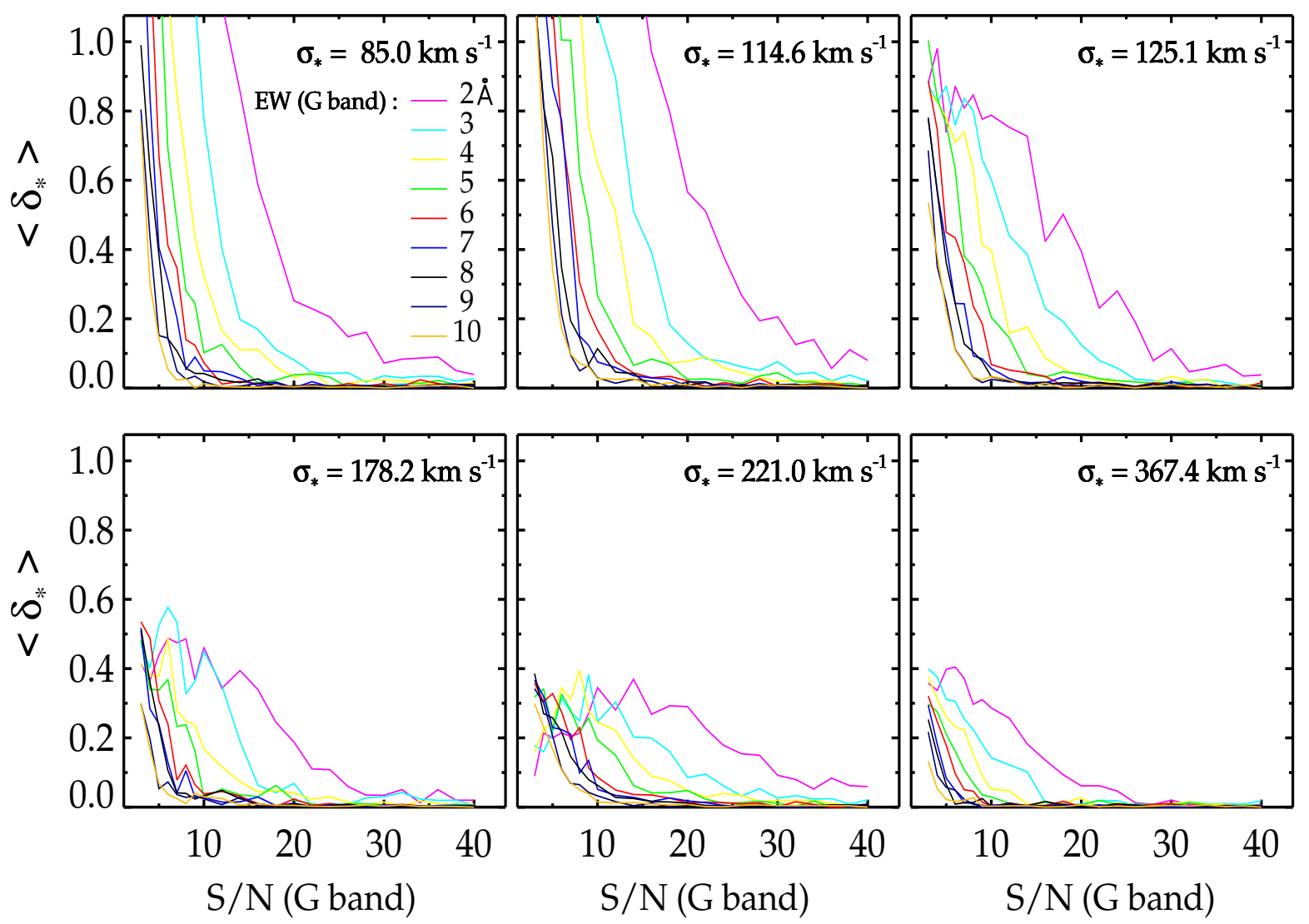

Figure 5. Systematic errors on the measured stellar velocity dispersions introduced by S/N and strength (EW) of the G band stellar feature. The panels show simulations performed on six high-S/N ( $\gtrsim 90)$ SDSS galaxy spectra with $\sigma_{*}=85,114,125,178,220$, and 360 $\mathrm{km} \mathrm{s}^{-1}$. The mean fractional error on $\sigma_{*}$ is given as $\left\langle\delta \sigma_{*}\right\rangle=\left\langle\left[\sigma_{*}\right.\right.$ (input) $-\sigma_{*}$ (output)] $/ \sigma_{*}($ input $\left.)\right\rangle$, for different combinations of $\mathrm{S} / \mathrm{N}$ and $\mathrm{EW}(\mathrm{G}$ band). 


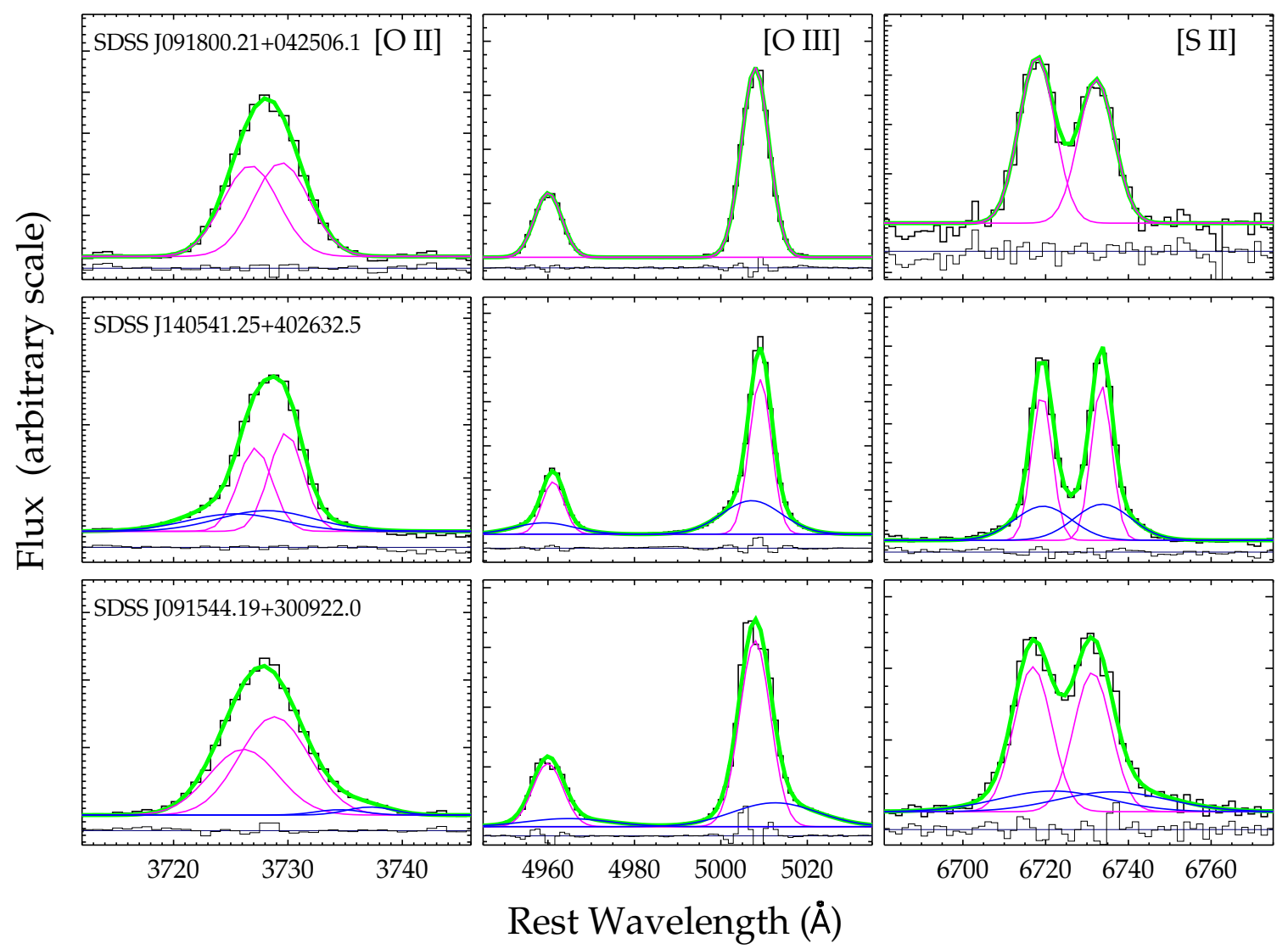

Figure 6. Sample fits for the emission lines (left) [O II] $\lambda \lambda 3726,3729$, (middle) [O III] $\lambda \lambda 4959,5007$, and (right) [S II] $\lambda \lambda 6716,6731$. The top object requires only a single Gaussian component for each line, whereas the lines for the other two objects each require two components, one whose wing is blueshifted (middle row) and the other that is redshifted (bottom row). The data are plotted in black, the best-fit model in green, and individual components in magenta (core) and blue (wing). The residuals (data minus model) are plotted on the bottom of each panel as thin black histograms.
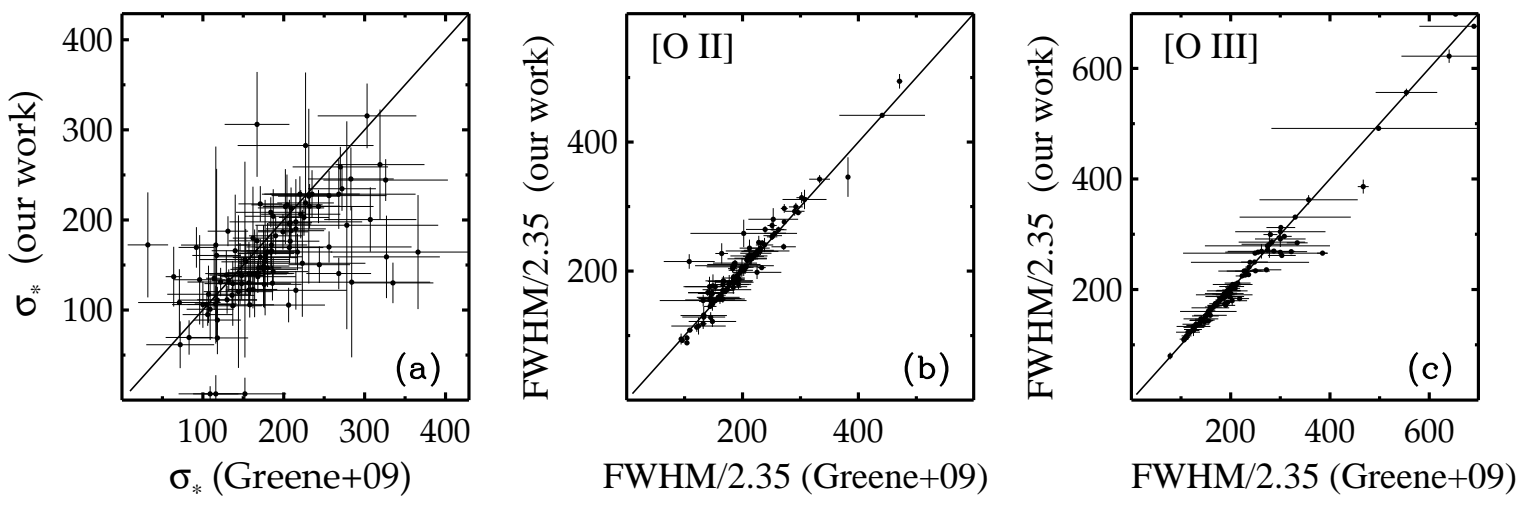

Figure 7. Comparison of our measurements with those of Greene et al. (2009) for (a) $\sigma_{*}$, (b) FWHM of [O II], and (c) FWHM of [O III]. The solid line denotes the 1:1 relation. 


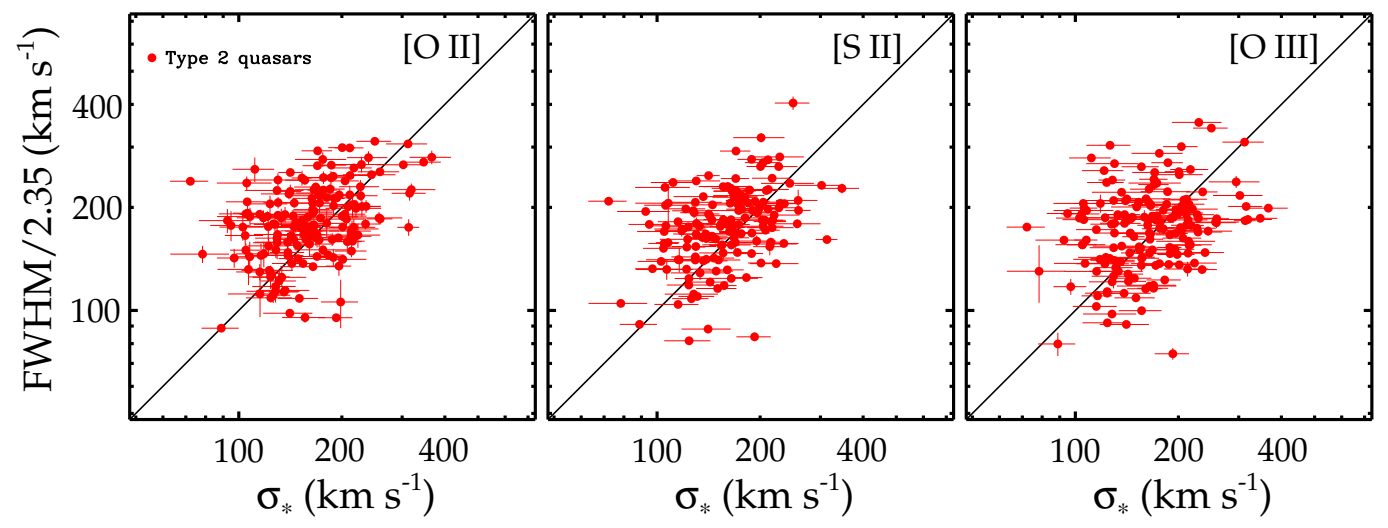

Figure 8. Comparison of stellar velocity dispersions $\sigma_{*}$ with gaseous velocity dispersions estimated from FWHM/2.35 of the [O II], [S II], and $[\mathrm{O}$ III] narrow emission lines. The data consist of the subset of type 2 quasars in this study having velocity dispersions measurements with fractional uncertainties $\leq 20 \%$. The solid line denotes the 1:1 relation. 

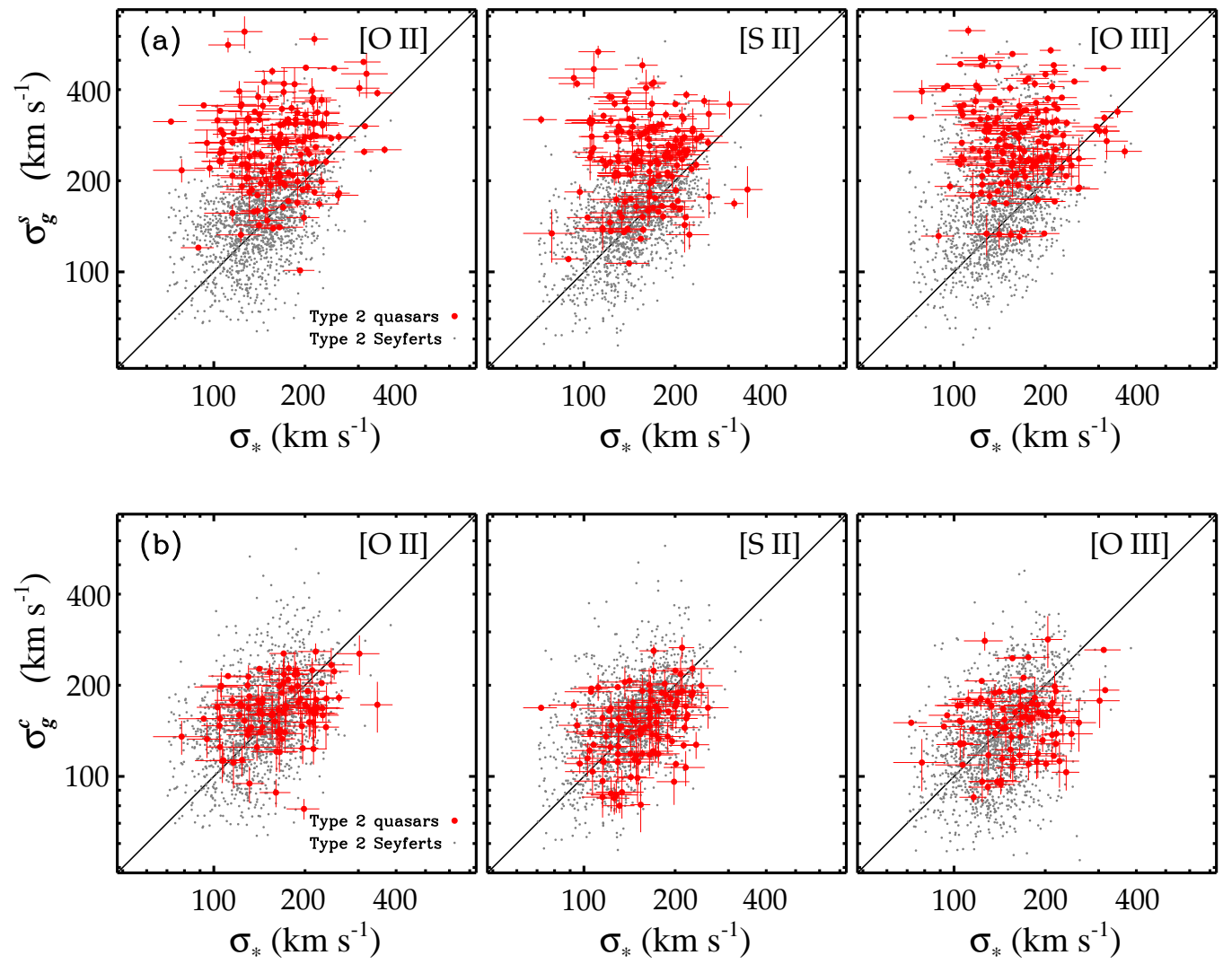

Figure 9. Comparison of stellar velocity dispersions $\sigma_{*}$ with gaseous velocity dispersions for the (left) [O II], (middle) [S II], and (right) [O III] emission lines measured from (a) the second moment $\left(\sigma_{g}^{s}\right)$ and (b) the core $\left(\sigma_{g}^{c}\right)$ of the line profile. The small grey points are from the lower luminosity type 2 AGNs (Seyferts) from Greene \& Ho (2005a). The red points with error bars are the subset of type 2 quasars in this study with fractional uncertainties $\leq 20 \%$. The solid line denotes the 1:1 relation. 

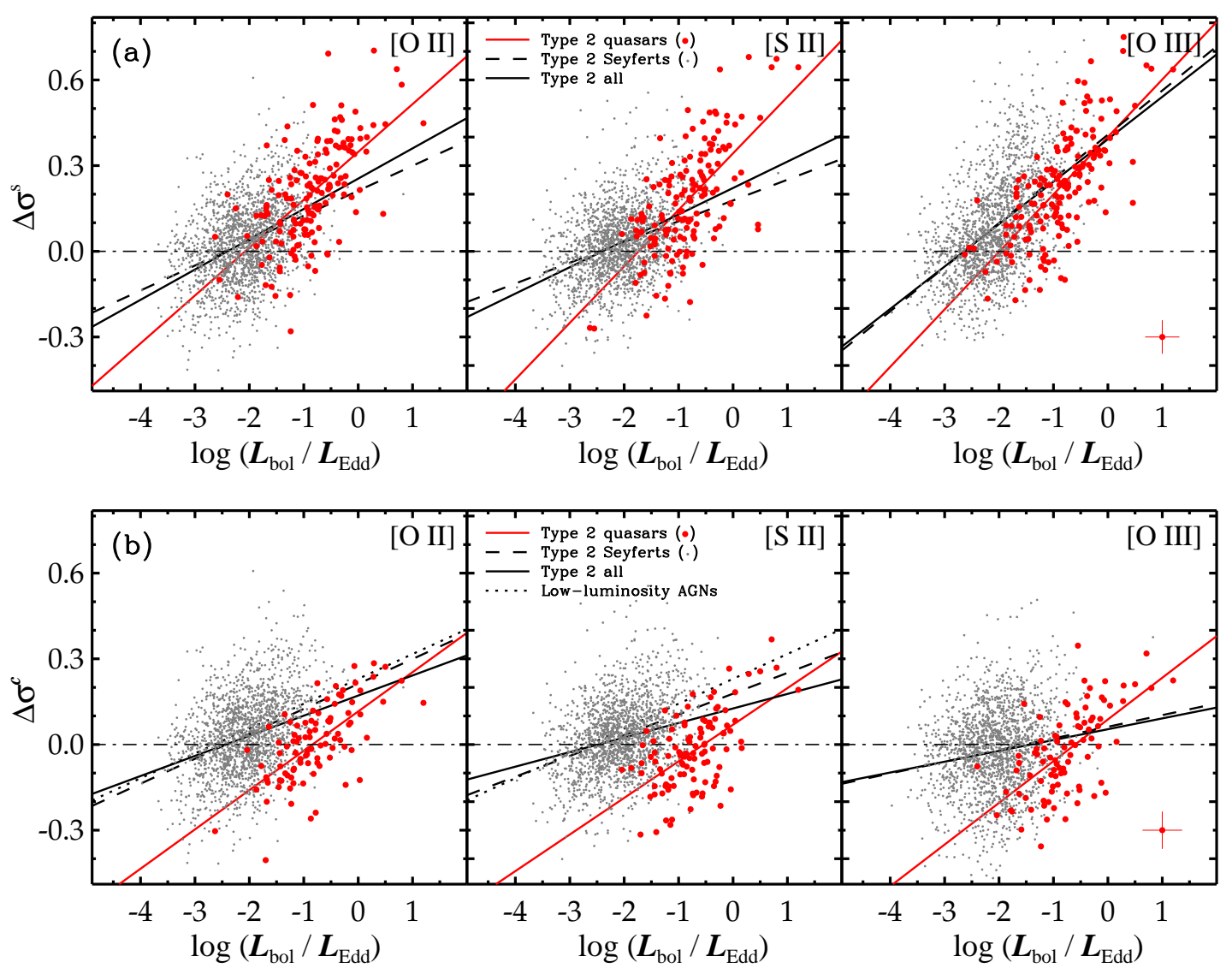

Figure 10. The relation between $L_{\mathrm{bol}} / L_{\mathrm{Edd}}$ and (a) $\Delta \sigma^{s} \equiv \log \sigma_{g}^{s}-\log \sigma_{*}$ and (b) $\Delta \sigma^{c} \equiv \log \sigma_{g}^{c}-\log \sigma_{*}$ for (left) [O II], (middle) [S II], and (right) [O III]. The small grey points are the lower luminosity type 2 Seyferts from Greene \& Ho (2005a). The red points are the subset of type 2 quasars in this study with fractional uncertainties $\leq 20 \%$; individual error bars are omitted for clarity, but a representative error bar is shown on the bottom-right corner of the [O III] plot. Best-fit relations, calculated using an ordinary least-squares regression with $L_{\mathrm{bol}} / L_{\mathrm{Edd}}$ as the independent variable, are given for the type 2 quasars (solid red line), type 2 Seyferts (dashed black line), and type 2 Seyferts and quasars combined (solid black line). For comparison, the relation derived by Ho (2009; his Equation 3) for [N II] $\lambda 6583$ is overplotted as a dotted black line in the $[\mathrm{O}$ II] and $[\mathrm{S} \mathrm{II}]$ plots of panel (b). 

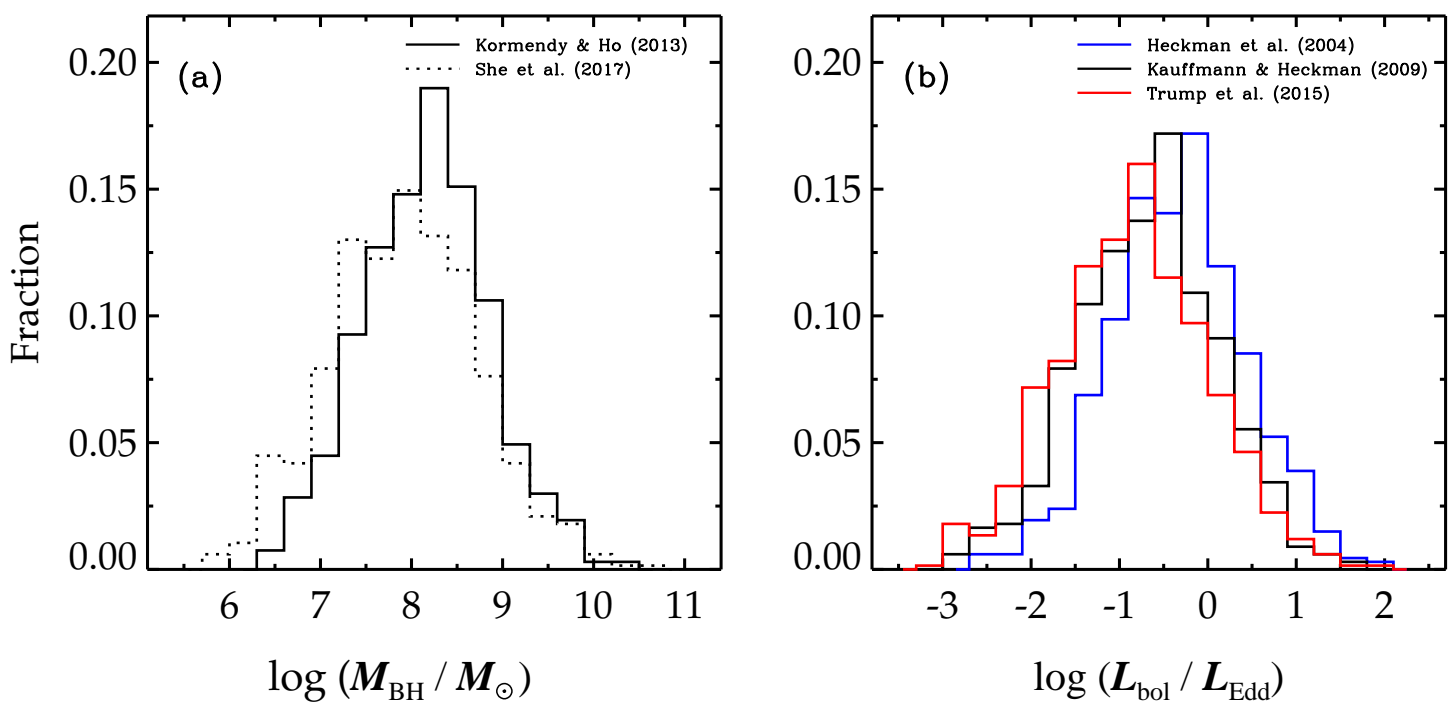

Figure 11. (a) Distribution of BH masses for our sample of type 2 quasars. The solid histograms assume the $M_{\mathrm{BH}}-\sigma_{*}$ relation for classical bulges and elliptical galaxies (Equation 2; Kormendy \& Ho 2013); the dotted histograms use the $M_{\mathrm{BH}}-\sigma_{*}$ relation for all bulge types (see footnote 7; She et al. 2017). (b) Distribution of Eddington ratios assuming the $M_{\mathrm{BH}}-\sigma_{*}$ relation for classical bulges and elliptical galaxies and the [O III] bolometric correction of Heckman et al. (2004; blue), Kauffmann \& Heckman (2009; black), and Trump et al. (2015; red), which is based on a power-law fit to the data from Lamastra et al. (2009).

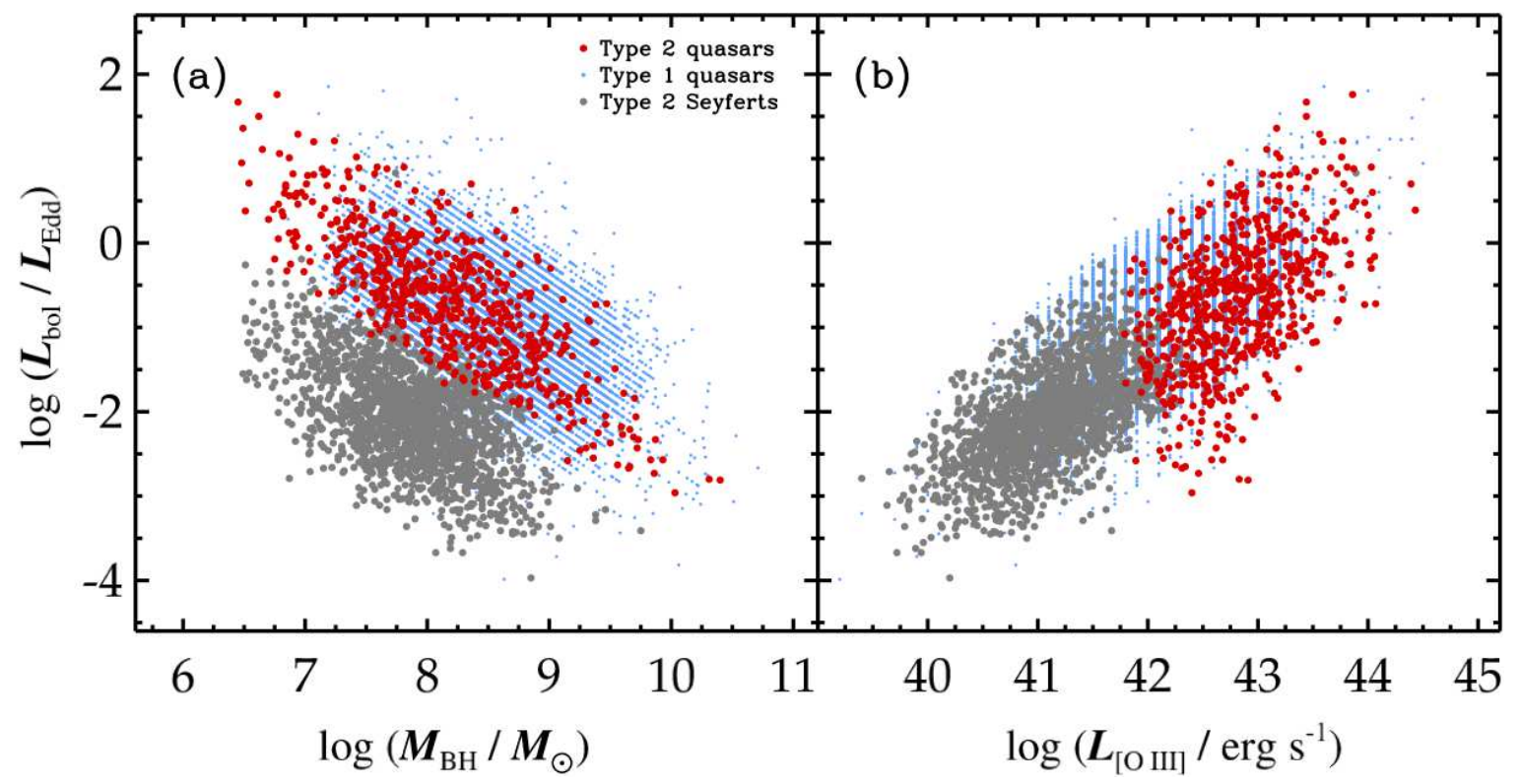

Figure 12. Distribution of Eddington ratio versus (a) BH mass and (b) [O III] luminosity for our sample of type 2 quasars (red points), lower luminosity, lower redshift type 2 Seyferts from Greene \& Ho (2005a; grey points), and type 1 quasars with $z<0.83$ from Shen et al. (2011; blue points). 